ESAIM: PROCEEDINGS, December 2012, Vol. 38, p. 135-162

F. Coquel, M. Gutnic, P. Helluy, F. Lagoutière, C. Rohde, N. Seguin, Editors

\title{
EXTENSION OF CENTERED HYDRODYNAMICAL SCHEMES TO UNSTRUCTURED DEFORMING CONICAL MESHES: THE CASE OF CIRCLES
}

\author{
Aude Bernard-Champmartin ${ }^{1}$, Erwan Deriaz ${ }^{2}$, Philippe Hoch*3 $^{* 3}$, Gerald \\ SAMBA $^{4}$ AND Michael SChAEFER ${ }^{5}$
}

\begin{abstract}
In a prior work [CEMRACS10], a curvilinear bi-dimensional finite volume extension of Lagrangian centered schemes GLACE [GLACE] on unstructured cells, whose edges are parameterized by rational quadratic Bézier curves was proposed and we showed numerical results for this scheme. Now, we extend the EUCCLHYD scheme [EUCCLHYD] to these cells. To simulate flows with evolving large deformations, we write a formalism allowing the time evolution of the conic parameter. As an example, this allows an edge changing from an ellipse segment to a hyperbolic one. In this framework, we consider the case of a mesh whose edges are circle segments with non fixed centers. We show that this formalism extends also the previous work [GLACE CIRCLE] (which is equivalent to [CEMRACS10] when conic edges are all circles). This is a necessary first step toward general conical deformation.
\end{abstract}

Résumé. Dans un travail précédent [CEMRACS10], une extension volume fini bi-dimensionnelle curviligne du schéma Lagrangien centré GLACE [GLACE] sur des cellules non structurées dont les bords sont paramétrés par des courbes de Bézier quadratiques rationnelles a été proposée. Maintenant, nous proposons une extension du schéma EUCCLHYD [EUCCLHYD] à ce type de cellules et pour lequel nous montrons quelques résultats numériques. Pour pouvoir suivre un écoulement subissant de grandes variations de forme, nous écrivons un formalisme qui permet l'évolution en temps du paramètre de chaque conique. Ainsi, un bord de maille peut d'un segment d'ellipse devenir en cours de calcul un segment d'hyperbole. Dans ce cadre, nous considérons le cas particulier d'un maillage dont les bords de cellules sont des arcs de cercle avec des centres non fixes. Nous montrons que ce formalisme constitue également une extension directe d'un travail précédent [GLACE CIRCLE] (qui équivaut à [CEMRACS10] dans le cas où les bords coniques sont tous des cercles). Ce résultat constitue une étape préliminaire nécessaire au cas des déformations coniques quelconques.

\section{INTRODUCTION}

The prototype system of interest is the Euler system written in flux form. The conservations of volume, density $\rho$, momentum $\rho \mathbf{U}$ and total energy $\rho E$ on a generic cell $C(t)$ write (see [DUVAUT] [EQU] [GLACE2D] [EUCCLHYD] [HDRPHMAIRE]):

${ }^{1}$ CMLA, ENS Cachan / LRC MESO, ENS Cachan and CEA, DAM, DIF, 61 av du Président Wilson, 94235 Cachan Cedex, France.

2 M2P2 - UMR-6181 CNRS IMT La Jetée Technopôle de Château-Gombert, 38 Rue Frédéric Joliot-Curie, 13451 MARSEILLE Cedex 20, France

$3 *$ Corresponding author: CEA, DAM, DIF, Bruyères-le-Chatel, F-91297 Arpajon Cedex, France

${ }^{4}$ CEA, DAM, DIF, Bruyères-le-Chatel, F-91297 Arpajon Cedex, France

${ }^{5}$ University of Muenster, Orléans-Ring 10, 48149 Münster, Germany

(C) EDP Sciences, SMAI 2012 


$$
\left\{\begin{array}{l}
D_{t} \int_{C(t)} 1 d v-\int_{\partial C(t)} \mathbf{U} \cdot \hat{\mathbf{N}} d s=0, \\
D_{t} \int_{C(t)} \rho d v=0, \\
D_{t} \int_{C(t)} \rho \mathbf{U} d v+\int_{\partial C(t)} P \hat{\mathbf{N}} d s=0, \\
D_{t} \int_{C(t)} \rho E d v+\int_{\partial C(t)} P \mathbf{U} \cdot \hat{\mathbf{N}} d s=0 .
\end{array}\right.
$$

We recall that (1) is the semi-Lagrangian (or updated Lagrangian) form of Euler system, where all the spatial quantities are written with Eulerian coordinates. It corresponds to a local differential operator at point $(\mathrm{t}, \mathrm{x})$ (x Eulerian coordinate):

$$
\begin{aligned}
& d_{t} \phi=\frac{\partial \phi}{\partial t}+\mathbf{U} \cdot \nabla \phi \\
& d_{t}(\phi J)=J\left(\frac{\partial \phi}{\partial t}+\nabla \cdot(\phi \mathbf{U})\right), \\
& \mathrm{J} \text { determinant of the Jacobian of Euler/Lagrange transformation. } \\
& D_{t} \int_{C(t)} \phi \mathrm{dx}=\int_{C(0)}\left(J\left(d_{t} \phi\right)+\phi \partial_{t} J\right) \mathrm{dX}, \quad \mathrm{X} \text { Lagrangian coordinate. }
\end{aligned}
$$

Generally such systems are discretized on polygonal type cells. However, some problems involve accurate geometric description such as curved boundary of the domain itself, discretization of a circular initial condition. Of course, it exists also non-stationary problems that will certainly be better approximated if the geometry of control volumes allows non null curvature (circles for symmetries on polar radial flows, interface curve between two materials, multi-material interfaces in mixed cells, etc...).

We want to extend this finite volume formalism (and then obtain higher genericity in geometric modeling) to cells with weaker hypothesis on edge representation. Generally, for cells with three or four edges, the curvilinear edges parameterization can be obtained by high order finite element such as $P_{2}, Q_{2}$ for Lagrangian higher order (at least third order) hydrodynamics; see [SHUSHEN, RIB] and also [FEV] for generical finite element method at the edges. Inside NURBS (Non Uniform Rational B-Spline) isogeometric analysis, we found that quadratic rational Bézier curves can exactly handle conic segments which include P1, Q1, P2 and Q2 edge's isoparametric representations. In particular the latter may not exactly match circles. In our case, we deal with arbitrary number of edges per cells and a fully explicit local finite volume framework. We emphasize that all our schemes do not need global matrix inversion contrary to most of above mentioned

schemes. The paper is organized as follows, in the first section we recall some essential description and geometric properties of the quadratic rational Bézier curves that are used in other sections. Specially, we recall the exact flux formula in [WAGUSEDE, WAGU] used to compute the area of a cell with an arbitrary number of conic edges, we recall that the analytic formula is just a function of the weight $-\omega$ - of the associated control point, this weight $\omega$ is also a caracterization of the conic type (straight line $\omega=0$, ellipse $0<\omega<1$, parabola $\omega=1$ or hyperbola $\omega>1$ ). We show that we can obtain the same kind of formula with a point on the edge parameterized by $q$ in $] 0,1[$ instead of the control point. We then reinterpret the area in terms of nodal/control point contributions as in GLACE or EUCCLHYD formalism. In the second section, we first write the $\omega$-EUCCLHYD scheme that extends the polygonal version [EUCCLHYD] and compare it with the $\omega$-GLACE version [CEMRACS10]. The $\omega$ version of each scheme continuously tends to original (polygonal) ones when $\omega$ tends to 0 . Secondly, we reformulate both schemes with new degrees of freedom located on the edges: $(\omega, q)$-GLACE/EUCCLHYD schemes. In the third section, we write the time derivative of the area with a varying $\omega$ parameter. We consider the special case of circle edges whose centers move in time. We then derive the associated $(\omega, q=1 / 2)$-GLACE scheme.

\section{Computing exaCt AREA FOR ARBitrary CONiC CELls}

In this part, we want to obtain an exact formula for the computation of area for arbitrary polygonal curved cells with any edge number. This is of course the first step to build any finite volume method. Let $\mathbf{X}$ in $\mathbb{R}^{2}$ be the position of a point inside generic cell $\bar{C}(t)(\bar{C}=C \cup \partial C)$, then

$$
|C(t)|=\int_{C(t)} 1 d v=\int_{C(t)} \frac{1}{2} \operatorname{div} \mathbf{X} d v=\frac{1}{2} \int_{\partial C(t)} \mathbf{X} . \hat{\mathbf{N}} d s, \hat{\mathbf{N}} \text { unit outward normal, } s \text { curvilinear abscissa }
$$


where by definition $s$ verifies $\left|\frac{d}{d s} \mathbf{X}\right|=1$ in (3). In the case where $\partial C(t)$ can be continuously decomposed into ne edges, each one being parameterized by a curve $q \in[0,1] \mapsto \mathbf{X}^{e}(q, t) \in \mathbb{R}^{2}$, we impose the continuity $\mathbf{X}^{e}(1, t)=\mathbf{X}^{e+1}(0, t)$ (cell's boundary is closed $\left.\mathbf{X}^{n e}(1, t)=\mathbf{X}^{1}(0, t)\right)$ and the orientation to be trigonometric. In the general case, we do not have a normal parameterization, but to get an admissible one, we must have $\left|\frac{d}{d q} \mathbf{X}\right| \neq 0$ everywhere on the curve. For this re-parameterization (from $s$ to $q$ ), formula (3) then writes:

$$
|C(t)|=\frac{1}{2} \sum_{e=1}^{n e}\left(\int_{0}^{1} \mathbf{X}^{e} \cdot\left(\frac{d}{d q} \mathbf{X}^{e}\right)^{\perp} d q\right), \quad \text { where }(a, b)^{\perp}:=(b,-a)
$$

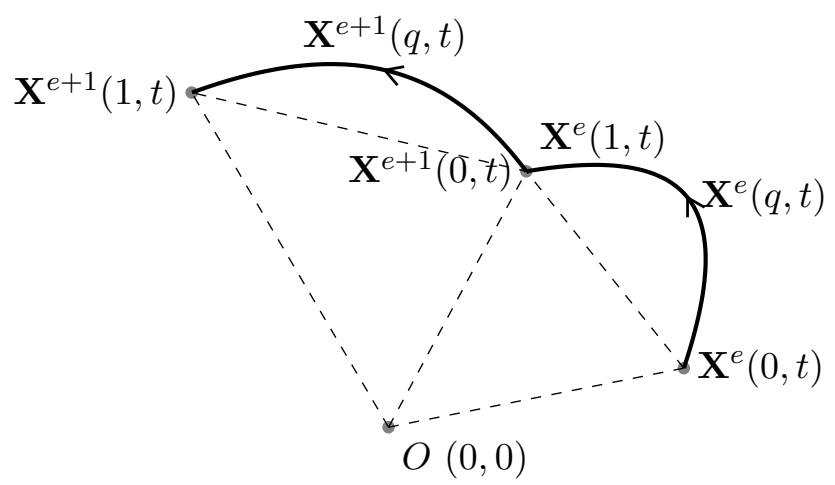

FiguRE 1. Decomposition of boundary integral for area computation.

We emphasize that at this level, our constraints are the following:

1) compute exactly this integral for a parametrization including straight polygonal, circular and parabolic cells as special cases.

2) obtain a formula involving the control points in an explicit analytical form to get a [GLACE]-like formalism.

We now describe the parametrization that fulfills these two constraints.

\subsection{Rational quadratic-Bézier parametrization}

For computer graphic and applied geometry communities, it is a well known result that any conic segment can be expressed by a rational quadratic Bézier curve, which is a special kind of NURBS (Non Uniform Rational B-Spline). Curved segment of any conic section can be represented by this kind of parametrization (see Figure 2 left):

$$
M^{\omega}(q)=\frac{M_{0}(1-q)^{2}+M_{1} 2 \omega q(1-q)+M_{2} q^{2}}{(1-q)^{2}+2 \omega q(1-q)+q^{2}}, \quad q \in[0,1] .
$$

The two parameters $\left(M_{1}, \omega\right)$ are the control point and its associated weight (here $\omega \geq 0$ ) for a given logical edge $\left(M_{0}, M_{2}\right)$.

We recall some properties presented in [LIGACH], that we will use in the following sections:

(1) The control simplex $\left(M_{0}, M_{1}, M_{2}\right)$ is not degenerate if and only if $M_{1}$ is not aligned to logical edge $\left(M_{0}, M_{2}\right)$.

(2) Convex hull: the curved segment $\left\{M^{\omega}(q), q \in[0,1]\right\}$ lies inside the control simplex for $\omega \geq 0$.

(3) Endpoints interpolation:

$$
\begin{aligned}
& M^{\omega}(0)=M_{0}, \quad M^{\omega}(1) \quad=\quad M_{2}, \\
& \frac{d}{d q} M^{\omega}(0)=2 \omega \mathbf{M}_{\mathbf{0}} \mathbf{M}_{\mathbf{1}}, \quad \frac{d}{d q} M^{\omega}(1)=2 \omega \mathbf{M}_{\mathbf{1}} \mathbf{M}_{\mathbf{2}} \text {. }
\end{aligned}
$$



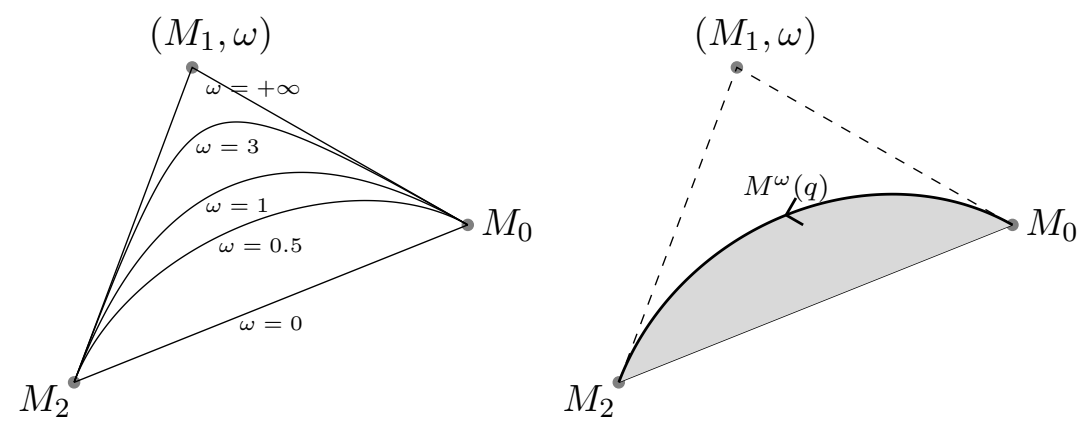

FiguRE 2. Left: Evolution of parametrization with respect to positive values of the parameter $\omega$ (all conic curves can be continuously represented), see property (4) below. Right: Conic section representation with rational quadratic-Bézier parametrization for edges, filled region needs to be exactly computed.

Hence $M^{\omega}(q)$ passes through $M_{0}$ and $M_{2}$ and for $\omega>0$ the two straight lines passing through these points respectively with directions $\frac{d}{d q} M^{\omega}(0)$ and $\frac{d}{d q} M^{\omega}(1)$ intersect at the control point $M_{1}$.

(4) Conic type parameter, see Figure 2 left:

(a) $\omega=0:$ degenerate straight segment.

(b) $0<\omega<1$ : ellipse segment, and in the case where $M_{1}$ is on the perpendicular bisector of $\left(M_{0}, M_{2}\right)$ and when $\omega:=\cos \left(\frac{\theta}{2}\right)$, circle segment is recovered, see Figure 3

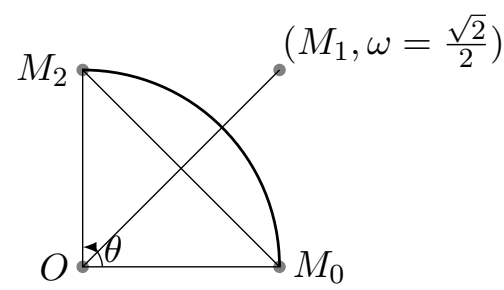

Figure 3. Circle recovered by special values of the couple $\left(M_{1}, \omega\right)$, if $O$ is the local "origin" attached to edge : $O M_{1}=\frac{R}{\omega}\left(\cos \left(\frac{\theta}{2}\right), \sin \left(\frac{\theta}{2}\right)\right), R=\left|O M_{0}\right|=\left|O M_{2}\right|$.

(c) $\omega=1$ : parabolic segment.

(d) $\omega>1$ : hyperbolic segment, and when $\omega \rightarrow \infty$ the conic segment tends to go through $M_{1}$.

(5) Shoulder point, see Figure 4: $S:=M^{\omega}(q=1 / 2)$ is the middle point of the curved segment, it can also be computed by:

$$
S=\frac{1}{2}\left(Q_{0}+Q_{2}\right), \text { where } \quad Q_{0}=\frac{1}{1+\omega}\left(M_{0}+\omega M_{1}\right), \quad Q_{2}=\frac{1}{1+\omega}\left(\omega M_{1}+M_{2}\right) .
$$

The tangent line of $M^{\omega}(q)$ at $S$ passes through $Q_{0}$ and $Q_{2}$ and is parallel to $\left(M_{0}, M_{2}\right)$, moreover $S$ is the point of the curve that maximizes the distance between the curve and $\left(M_{0}, M_{2}\right)$.

(6) Implicit form: the implicit equation for $M^{\omega}(q)=\left(x^{\omega}(q), y^{\omega}(q)\right)$ writes

$$
\lambda_{1}(x, y)^{2}-4 \omega^{2} \lambda_{0}(x, y) \lambda_{2}(x, y)=0
$$

where $\left\{\lambda_{i}(x, y)\right\}_{i=0,1,2}$ correspond to the barycentric coordinates of the point $(x, y)$ with respect to the control simplex $\left(M_{0}, M_{1}, M_{2}\right)$. 


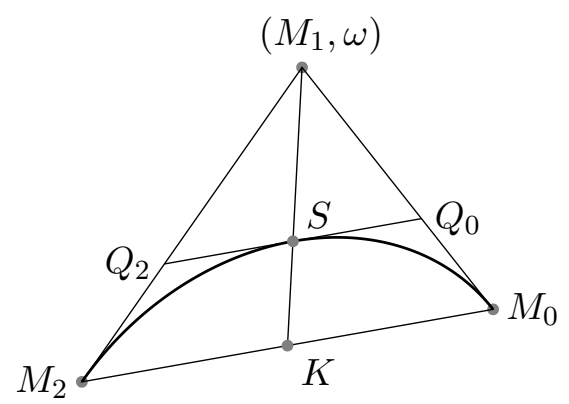

Figure 4. Shoulder point of rational quadratic Bézier curve (5).

\subsection{Edge based formula}

Computing (4) with rational quadratic Bézier parametrization, for only one segment in (5) we have:

$$
I=\frac{1}{2} \int_{0}^{1}\left(x^{\omega}(q) \frac{d}{d q} y^{\omega}(q)-\frac{d}{d q} x^{\omega}(q) y^{\omega}(q)\right) d q
$$

where $\left(x^{\omega}(q), y^{\omega}(q)\right)$ are the cartesian coordinates of the point $M^{\omega}(q)$. This integral can be decomposed in two parts (see Figure 1):

(1) the area between the curve $M^{\omega}(q)$ and the segment $\left(M_{0}, M_{2}\right)$ (see Figure 2 right). We denote $A\left(M^{\omega}(q), M_{0}, M_{1}, M_{2}\right)$ this (exact) area and $A^{T}\left(M_{0}, M_{1}, M_{2}\right)$ the (exact) area of the straight edges control simplex.

(2) the area of the simplex $\left(\mathrm{O}, M_{0}, M_{2}\right)$ with straight edges: $A^{T}\left(O, M_{0}, M_{2}\right)$.

In [WAGU], the author gives a way to compute it when $M_{0}=(0,0)$ and $M_{2}=\left(x_{2}, 0\right)$ so that we need to add this term to compute the integral (9), hence for arbitrary $M_{0}$ and $M_{2}$ :

$$
I=A\left(M^{\omega}(q), M_{0}, M_{1}, M_{2}\right)+A^{T}\left(O, M_{0}, M_{2}\right)=A\left(M^{\omega}(q), M_{0}, M_{1}, M_{2}\right)+\frac{1}{2} \operatorname{det}\left(\mathbf{O M}_{\mathbf{0}}, \mathbf{O M}_{\mathbf{2}}\right) .
$$

We now recall the results in [WAGU] to compute $A\left(M^{\omega}(q), M_{0}, M_{1}, M_{2}\right)$. For each kind of conic $(\omega=0$ to $\omega \rightarrow \infty)$, there exists a function $f(\omega)$ such that:

$$
A\left(M^{\omega}(q), M_{0}, M_{1}, M_{2}\right)=f(\omega) A^{T}\left(M_{0}, M_{1}, M_{2}\right) .
$$

We recall $f(\omega)$ (see Figure 5 ) is a continuous strictly increasing function on $[0, \infty[$ with value in $[0,1[$.

$$
\left\{\begin{array}{l}
\text { (1) if } \omega=0, \text { then } f(\omega)=0, \\
\text { (2) if } 0<\omega<1, \text { then } f(\omega)=\frac{2 \omega}{1-\omega^{2}}\left(\frac{1}{\sqrt{1-\omega^{2}}} \operatorname{atan}\left(\sqrt{\frac{1-\omega}{1+\omega}}\right)-\frac{\omega}{2}\right), \\
\text { (3) if } \omega=1 \text {, then } f(\omega)=\frac{2}{3}, \\
\text { (4) if } \omega>1 \text {, then } f(\omega)=\frac{\omega}{\omega^{2}-1}\left(\omega+\frac{1}{\sqrt{\omega^{2}-1}} \log \left(\omega-\sqrt{\omega^{2}-1}\right)\right) .
\end{array}\right.
$$

Actually, we have much more regularity (see Figure 6):

\section{Remark 1.}

$$
\left\{\begin{array}{l}
\text { The function } f(\omega) \text { is of class } C^{1}([0, \infty[), \\
\lim _{w \rightarrow 1^{-}} f^{\prime}(w)=\frac{4}{15}=\lim _{w \rightarrow 1^{+}} f^{\prime}(w) .
\end{array}\right.
$$

In the following, we will need this result to take into account deformation. 

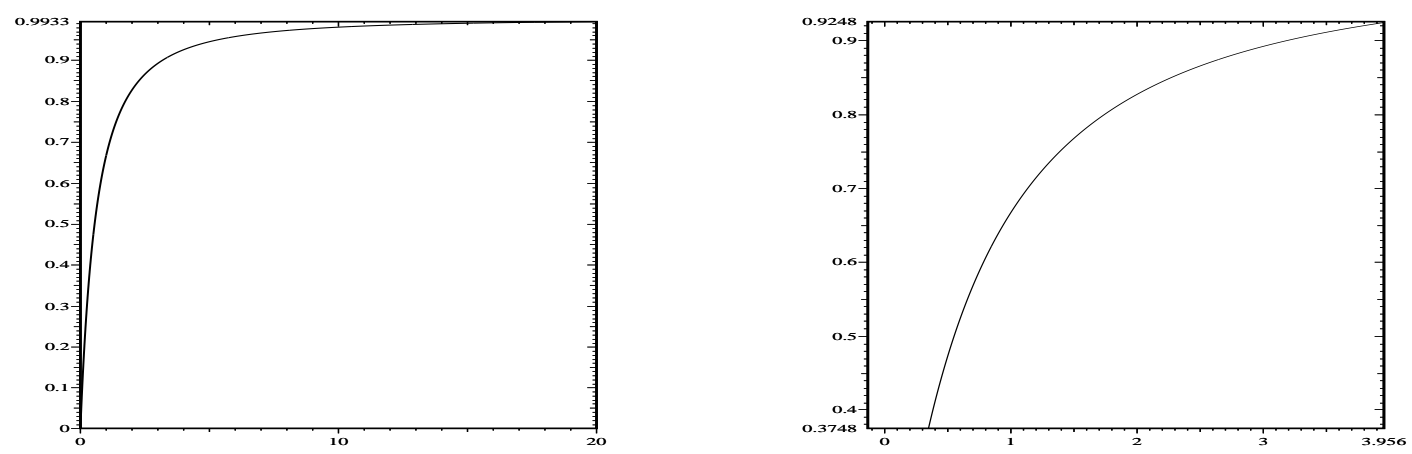

FiguRE 5. Left: graph of the nonlinear function $f(\omega)$ weighting the area of simplex $\left(M_{0}, M_{1}, M_{2}\right)$ in formula $(11)(12)$, in abscissa weight $\omega$. Right: zoom near $\omega=1,\left(f(1)=\frac{2}{3}\right)$.
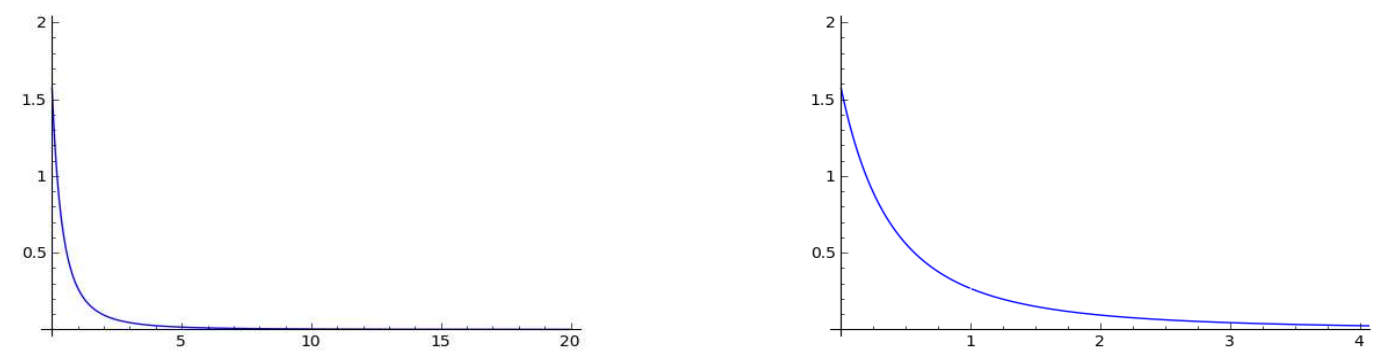

FiguRE 6. Left: graph of $f^{\prime}(\omega)$ (see also Figure 5), Right: zoom near $\omega=1,\left(f^{\prime}(1)=\frac{4}{15}\right.$ ).

\subsection{Expression of area formula with degrees of freedom based on arbitrary parameter value on edge: $M^{\omega}(q)$}

As noticed, the control point $M_{1}$ is not located on the conical edge, it may be interesting to obtain such degree of freedom located truly on the edge. To do so, we compute the (unique) intersection point of straight line $\left(M_{1}, M^{\omega}(q)\right)$ (see definition (5)), and the straight segment $\left[M_{0}, M_{2}\right]$ (see Figure 8):

$$
K(q)=\frac{1}{q^{2}+(1-q)^{2}}\left(q^{2} M_{2}+(1-q)^{2} M_{0}\right),
$$

which is a squared convex combination of the two extremities. We note that any point $M^{\omega}(q)$ (see 5 ) can be obtained:

$$
\begin{aligned}
& M^{\omega}(q)=(1-q) Q_{0}(q)+q Q_{2}(q), \quad \text { where } \\
& Q_{0}(q)=\frac{(1-q) M_{0}+\omega q M_{1}}{(1-q)^{2}+2 \omega q(1-q)+q^{2}}, \quad Q_{2}(q)=\frac{\omega(1-q) M_{1}+q M_{2}}{(1-q)^{2}+2 \omega q(1-q)+q^{2}}
\end{aligned}
$$

Now, we can establish the following relation:

$$
\left\{\begin{array}{l}
\mathbf{K}(\mathbf{q}) \mathbf{M}_{\mathbf{1}}=\alpha(\omega, q) \mathbf{K}(\mathbf{q}) \mathbf{M}^{\omega}(\mathbf{q}), \quad \text { where } \\
\alpha(\omega, q)=\frac{(1-q)^{2}+2 \omega q(1-q)+q^{2}}{2 \omega q(1-q)} .
\end{array}\right.
$$

We then obtain a one parameter formula to re-write the simplex control area in terms of any point on the curve: 


\section{Proposition 2.}

$$
A^{T}\left(M_{0}, M_{1}, M_{2}\right)=\alpha(\omega, q) A^{T}\left(M_{0}, M^{\omega}(q), M_{2}\right)
$$

Proof: The area ratio of two simplices $\left(M_{0}, M_{1}, M_{2}\right)$ and $\left(M_{0}, M^{\omega}(q), M_{2}\right)$ is nothing but the coefficient $\alpha(\omega, q)$ in (16) because two out of three points are the same.
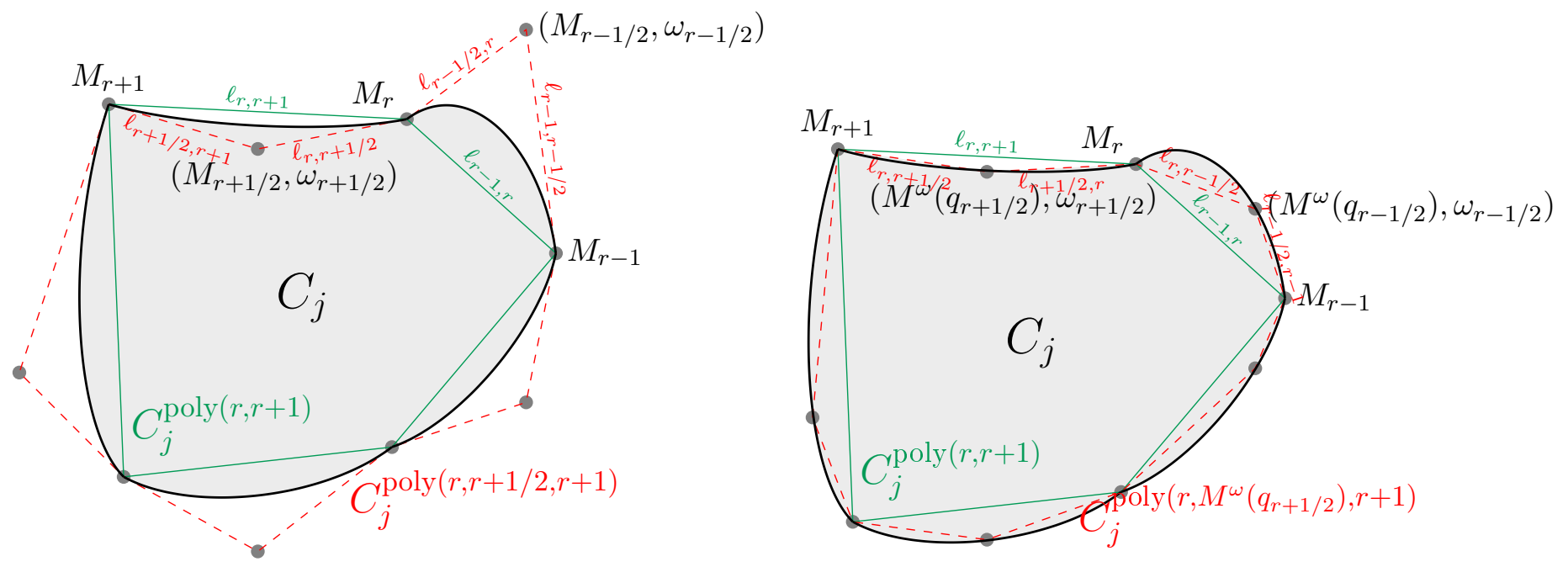

FiguRE 7. Left: Generic cell $C_{j}$ and its inclusion in the convex hull of logical polygonal with (in red dashed line) and without (in green polyline) control point (here $\ell_{a, b}$ is the euclidean distance between point $a$ and $b$ ), Right: The same cell is described by new position of degrees of freedom located on edges.

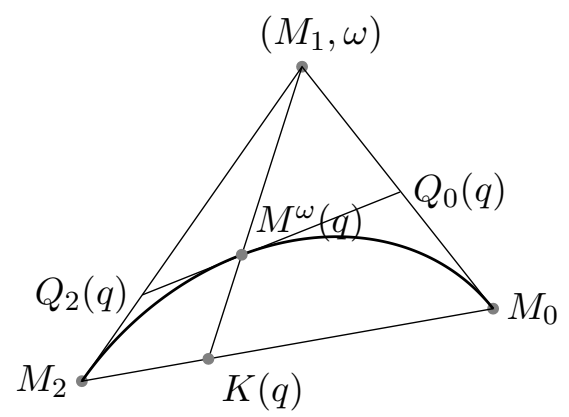

FiguRe 8. Arbitrary position of degree of freedom $M^{\omega}(q)$ for rational quadratic Bézier curve (5), notice that taking $\mathrm{q}=1 / 2$, we recover (Figure 4 ) (thanks to the one to one mapping between $M_{1}$ and $M^{\omega}(q)$ for $\left.q \in\right] 0,1[$ in (18) (19)).

We then obtain:

$$
A\left(M^{\omega}(q), M_{0}, M_{1}, M_{2}\right)=f(\omega) A^{T}\left(M_{0}, M_{1}, M_{2}\right)=g(\omega, q) A^{T}\left(M_{0}, M^{\omega}(q), M_{2}\right)
$$

with

$$
g(\omega, q)=f(\omega) \alpha(\omega, q) .
$$

Remark 3. - For fixed $q \in] 0,1\left[\right.$, the function $\omega \rightarrow g(\omega, q)$ is $C^{1}([0 ;+\infty[)$.

- This will allow us to adapt the location of the degrees of freedom in the original $\omega-G L A C E$ scheme, we are then able to deduce new geometrical vector $C_{j}^{r, \omega, q}$. 
- We recover the Shoulder point definition and classical parameters $\left(S, K, Q_{0}, Q_{1}\right)$ in Figure (4) by taking $q=\frac{1}{2}$ in (14) (16) see also Figure (4) and Figure (8):

$$
S=\frac{1}{2(1+\omega)}\left(M_{0}+2 \omega M_{1}+M_{2}\right), \quad K=\frac{1}{2}\left(M_{0}+M_{2}\right)
$$

and then

$$
\begin{aligned}
& A^{T}\left(M_{0}, M_{1}, M_{2}\right)=\frac{1+\omega}{\omega} A^{T}\left(M_{0}, S, M_{2}\right), \\
& A\left(M^{\omega}(q), M_{0}, M_{1}, M_{2}\right)=h(\omega) A^{T}\left(M_{0}, S, M_{2}\right) .
\end{aligned}
$$

with

$$
h(\omega)=f(\omega)\left(\frac{1+\omega}{\omega}\right),
$$

which can also be refound by using Thales theorem (because in this case $\left[Q_{0}, Q_{2}\right]$ is parallel to $\left.\left[M_{0}, M_{2}\right]\right)$.

\subsection{Nodal based formula}

In this part, we express the area formula of a cell $C_{j}$ in terms of two different groups of degrees of freedom:

(1) First, when using $M_{r}, M_{r+1}$ and the natural control point $\left(M_{r+1 / 2}, \omega_{r+1 / 2}\right)$ (see Figure (7) Left), we get the following see [CEMRACS10]:

$$
\left|C_{j}\right|=\frac{1}{2}\left(\sum_{r} \mathbf{C}_{j}^{r, \omega} \cdot \mathbf{O} \mathbf{M}_{r}+\sum_{r+1 / 2} \mathbf{C}_{j}^{r+1 / 2, \omega} \cdot \mathbf{O} \mathbf{M}_{r+1 / 2}\right):=\frac{1}{2} \sum_{\text {dof }} \mathbf{C}_{j}^{\mathrm{dof}, \omega} \cdot \mathbf{O M}_{\mathrm{dof}},
$$

where the expression of $\mathbf{C}_{j}^{r, \omega}$ and $\mathbf{C}_{j}^{r+1 / 2, \omega}$ are described below using notation and results of (12):

$$
\begin{gathered}
\mathbf{C}_{j}^{r, \omega}=\frac{1}{2}\left(\begin{array}{c}
\left(1-f\left(\omega_{r-1 / 2}\right)\right) \mathbf{N}_{r-1, r}+\left(1-f\left(\omega_{r+1 / 2}\right)\right) \mathbf{N}_{r, r+1} \\
\left.+f\left(\omega_{r-1 / 2}\right) \mathbf{N}_{r-1 / 2, r}+f\left(\omega_{r+1 / 2}\right) \mathbf{N}_{r, r+1 / 2}\right),
\end{array}\right. \\
\mathbf{C}_{j}^{r+1 / 2, \omega}=\frac{f\left(\omega_{r+1 / 2}\right)}{2}\left(\mathbf{N}_{r, r+1 / 2}+\mathbf{N}_{r+1 / 2, r+1}\right) .
\end{gathered}
$$

Moreover, we have the following identities:

$\left\{\begin{array}{l}\text { For each cell : } \sum_{r} \mathbf{C}_{j}^{r, \omega}+\sum_{r+1 / 2} \mathbf{C}_{j}^{r+1 / 2, \omega}=0, \\ \text { for each internal node }: \sum_{j} \mathbf{C}_{j}^{r, \omega}=0, \text { for two adjacent cells } \mathrm{j} \text { and } \mathrm{k}: \mathbf{C}_{j}^{r+1 / 2, \omega}+\mathbf{C}_{k}^{r+1 / 2, \omega}=0 .\end{array}\right.$

(2) Secondly, if we use $\left(M_{r}, M_{r+1}\right)$ and an arbitrary point on the curve $\left(M^{\omega}\left(q_{r+1 / 2}\right), \omega_{r+1 / 2}\right)$ for $q_{r+1 / 2} \in$ ]0,1[ fixed (see Figure (7) Right).

To simplify the notations, in the following, for fixed $q \in] 0,1[$ :

$$
\begin{aligned}
& \tilde{C}_{j}^{r, \omega}:=C_{j}^{r, \omega, q}, \\
& \tilde{C}_{j}^{r+1 / 2, \omega}:=C_{j}^{r+1 / 2, \omega, q}
\end{aligned}
$$

Note that by replacing $f(\omega)$ by $g(\omega, q)$ (see 19) in (23) and (24) and $N_{r, r+1 / 2}$ by $\tilde{N}_{r, r+1 / 2}$ where $\tilde{N}_{r, r+1 / 2}$ is the vector obtained by a $\left(\frac{\pi}{2}\right)$ rotation of $M_{r} M^{\omega}\left(q_{r+1 / 2}\right)$, we obtain a new formula of area expressed by edges contributions coming from endpoint nodes $M_{r}$ and other parameters:

$$
\left|C_{j}\right|=\frac{1}{2}\left(\sum_{r} \tilde{\mathbf{C}}_{j}^{r, \omega} \cdot \mathbf{O} \mathbf{M}_{r}+\sum_{r+1 / 2} \tilde{\mathbf{C}}_{j}^{r+1 / 2, \omega} \cdot \mathbf{O M}^{\omega}\left(\mathbf{q}_{\mathbf{r}+\mathbf{1} / \mathbf{2}}\right)\right):=\frac{1}{2} \sum_{\text {newdof }} \tilde{\mathbf{C}}_{j}^{\text {newdof }, \omega} \cdot \mathbf{O M}_{\text {newdof }}
$$




$$
\begin{gathered}
\tilde{\mathbf{C}}_{j}^{r, \omega}=\frac{1}{2}\left(\left(1-g\left(\omega_{r-1 / 2}, q_{r-1 / 2}\right)\right) \mathbf{N}_{r-1, r}+\left(1-g\left(\omega_{r+1 / 2}, q_{r+1 / 2}\right)\right) \mathbf{N}_{r, r+1}\right. \\
\left.+g\left(\omega_{r-1 / 2}, q_{r-1 / 2}\right) \tilde{\mathbf{N}}_{r-1 / 2, r}+g\left(\omega_{r+1 / 2}, q_{r+1 / 2}\right) \tilde{\mathbf{N}}_{r, r+1 / 2}\right) \\
\tilde{\mathbf{C}}_{j}^{r+1 / 2, \omega}=\frac{g\left(\omega_{r+1 / 2}, q_{r+1 / 2}\right)}{2}\left(\tilde{\mathbf{N}}_{r, r+1 / 2}+\tilde{\mathbf{N}}_{r+1 / 2, r+1}\right)
\end{gathered}
$$

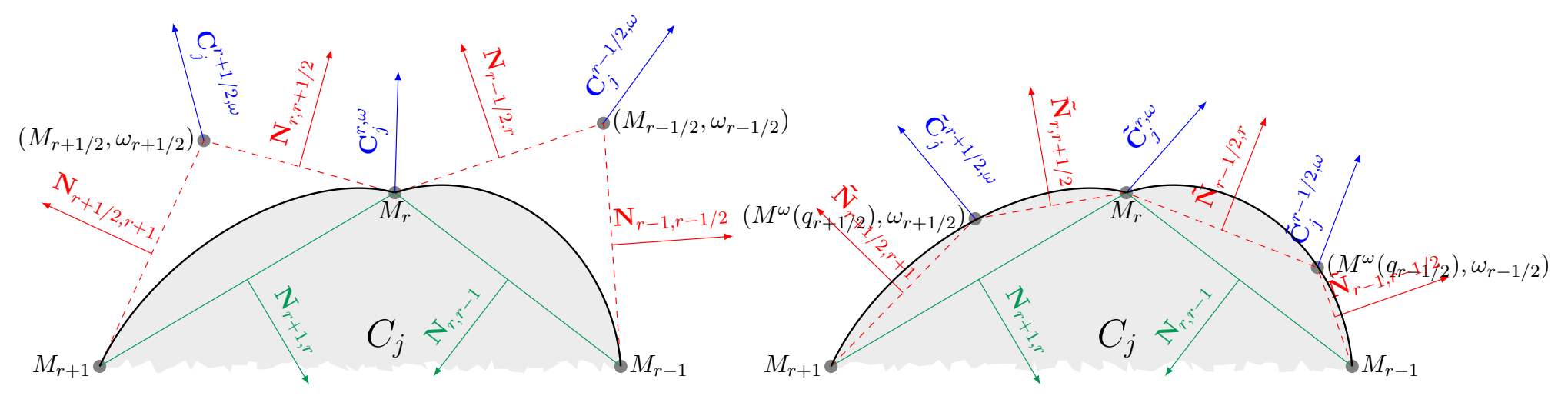

FiguRE 9. Left (resp. Right) decomposition of vector $\mathbf{C}_{j}^{\text {dof, } \omega}\left(\right.$ resp. $\left.\tilde{\mathbf{C}}_{j}^{\text {dof, }, \omega}\right)$ at degrees of freedom (for short: dof) for conic cells (in each case they are given by $\nabla_{M_{\mathrm{dof}}}\left|C_{j}\right|$ ). Left: degrees of freedom include control points $M_{r+1 / 2}$ (not on the curve in general), Right: degree of freedom that can be any point on the curve $M^{\omega}\left(q_{r+1 / 2}\right)$.

\section{2. $\omega$-EUCCLHYD AND $(\omega, q)-$ GLACE AND EUCCLHYD SCHEMES}

In this section, we want to present some extensions of the conical centered schemes GLACE and EUCCLHYD for the hydrodynamic equation (1):

$$
\left\{\begin{array}{l}
D_{t} \int_{C(t)} 1 d v-\int_{\partial C(t)} \mathbf{U} \cdot \hat{\mathbf{N}} d s=0 \\
D_{t} \int_{C(t)} \rho d v=0 \\
D_{t} \int_{C(t)} \rho \mathbf{U} d v+\int_{\partial C(t)} P \cdot \hat{\mathbf{N}} d s=0 \\
D_{t} \int_{C(t)} \rho E d v+\int_{\partial C(t)} P \mathbf{U} \cdot \hat{\mathbf{N}} d s=0
\end{array}\right.
$$

\section{1. $\omega$-EUCCLHYD scheme}

In this part, we present some extension of the polygonal EUCCLHYD [EUCCLHYD] scheme to curvilinear conical unstructured meshes. We take the following notations (see Figure (9) Left):

$$
\left\{\begin{array}{l}
\mathbf{N}_{j}^{r, \omega-}:=\left(1-f\left(\omega_{r-1 / 2}\right)\right) \mathbf{N}_{r-1, r}+f\left(\omega_{r-1 / 2}\right) \mathbf{N}_{r-1 / 2, r} \\
\mathbf{N}_{j}^{r, \omega+}:=\left(1-f\left(\omega_{r+1 / 2}\right)\right) \mathbf{N}_{r, r+1}+f\left(\omega_{r+1 / 2}\right) \mathbf{N}_{r, r+1 / 2} \\
\mathbf{N}_{j}^{r+1 / 2, \omega-}:=f\left(\omega_{r+1 / 2}\right) \mathbf{N}_{r, r+1 / 2} \\
\mathbf{N}_{j}^{r+1 / 2, \omega+}:=f\left(\omega_{r+1 / 2}\right) \mathbf{N}_{r+1 / 2, r+1}
\end{array}\right.
$$

Remark 4. (1) We have the following link between $C_{j}^{r, \omega}$ "GLACE vectors" (23) (24) see Figure (9) and "EUCCLHYD vectors" defined by (30):

$$
\left\{\begin{array}{l}
\mathbf{C}_{j}^{r, \omega}=\frac{1}{2}\left(\mathbf{N}_{j}^{r, \omega-}+\mathbf{N}_{j}^{r, \omega+}\right) \\
\mathbf{C}_{j}^{r+1 / 2, \omega}=\frac{1}{2}\left(\mathbf{N}_{j}^{r+1 / 2, \omega-}+\mathbf{N}_{j}^{r+1 / 2, \omega+}\right) .
\end{array}\right.
$$


(2) Moreover, we have the following identities:

$\left\{\begin{array}{l}\text { For each cell } C_{j}: \sum_{r}\left(\mathbf{N}_{j}^{r, \omega-}+\mathbf{N}_{j}^{r, \omega+}\right)+\sum_{r+1 / 2}\left(\mathbf{N}_{j}^{r+1 / 2, \omega-}+\mathbf{N}_{j}^{r+1 / 2, \omega-}\right)=0, \\ \text { for internal node } M_{r}: \sum_{j}\left(\mathbf{N}_{j}^{r, \omega-}+\mathbf{N}_{j}^{r, \omega+}\right)=0, \\ \text { and two adjacent cells } j \text { and } k: \mathbf{N}_{j}^{r+1 / 2, \omega-}+\mathbf{N}_{j}^{r+1 / 2, \omega+}+\mathbf{N}_{k}^{r+1 / 2, \omega-}+\mathbf{N}_{k}^{r+1 / 2, \omega+}=0 .\end{array}\right.$

This is a continuous $\omega$-EUCCLHYD formalism going from $\mathbf{C}_{j}^{\mathrm{poly}(r, r+1)}$ to $\mathbf{C}_{j}^{\mathrm{poly}(r, r+1 / 2, r+1)}$ cells, see Figure \%:

(a) For $\omega=0, \mathbf{N}_{j}^{r, \omega-}=\mathbf{N}_{r-1, r}, \mathbf{N}_{j}^{r, \omega+}=\mathbf{N}_{r, r+1}$ and $\mathbf{N}_{j}^{r+1 / 2, \omega \pm}=\mathbf{0}$, so that for the two first, we recover the classical normals of [EUCCLHYD] on polygonal cells $\mathbf{C}_{j}^{\text {poly }(r, r+1)}$.

(b) For $\omega \rightarrow \infty, \mathbf{N}_{j}^{r, \omega-}=\mathbf{N}_{r-1 / 2, r}, \mathbf{N}_{j}^{r, \omega+}=\mathbf{N}_{r, r+1 / 2}$ and $\mathbf{N}_{j}^{r+1 / 2, \omega-}=\mathbf{N}_{r-1 / 2, r}, \mathbf{N}_{j}^{r+1 / 2, \omega+}=$ $\mathbf{N}_{r+1 / 2, r+1}$, so that for both, we also recovered classical normals of [EUCCLHYD] on polygonal cells $\mathbf{C}_{j}^{\text {poly }(r, r+1 / 2, r+1)}$.

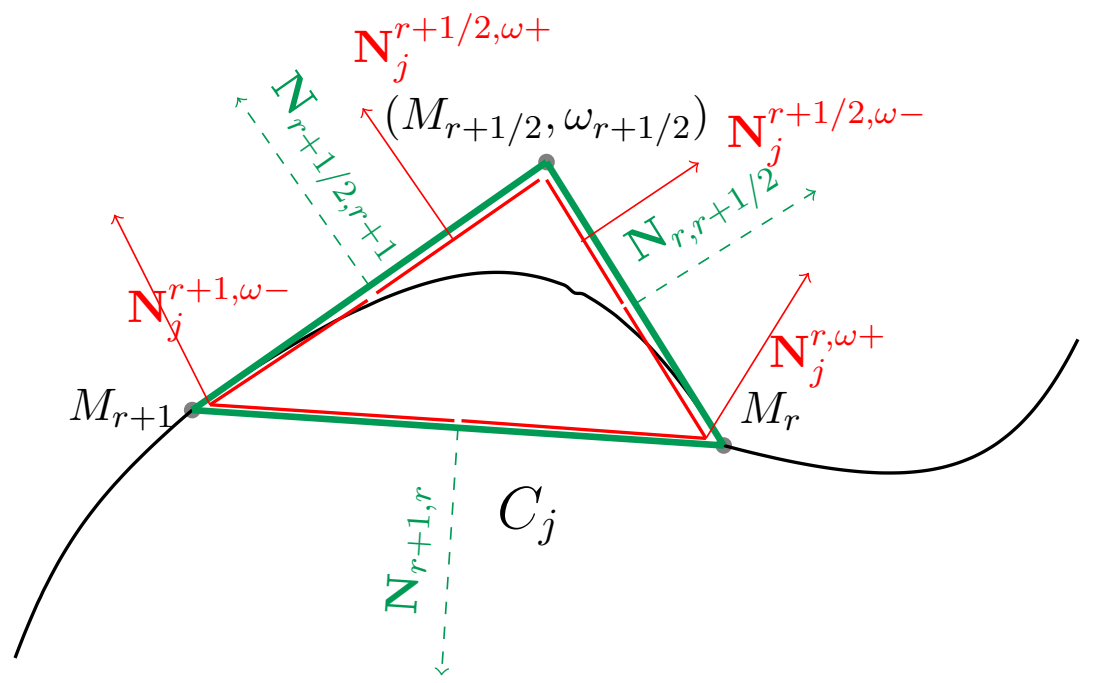

FiguRE 10. The four effective normals (red) $\mathbf{N}_{j}^{d o f, \omega \pm}$ per curved edge for the conical $\omega$-EUCCLHYD scheme, see relation (30) with geometric normals (green).

If we denote $M(t)$ the mass of cell $C(t)\left(M(t)=\int_{C(t)} \rho d v\right)$ which is constant, the semi-discrete $\omega$ EUCCLHYD scheme on conic cells writes:

$$
\left\{\begin{aligned}
M_{j} \tau_{j}^{\prime}(t)= & \sum_{r} \frac{1}{2}\left(\mathbf{N}_{j}^{r, \omega-}+\mathbf{N}_{j}^{r, \omega+}\right) \cdot \mathbf{U}_{r}+\sum_{r+1 / 2} \frac{1}{2}\left(\mathbf{N}_{j}^{r+1 / 2, \omega-}+\mathbf{N}_{j}^{r+1 / 2, \omega+}\right) \cdot \mathbf{U}_{r+1 / 2}, \tau_{j}(t)=\frac{1}{\rho_{j}(t)}, \\
M_{j} \mathbf{U}_{j}^{\prime}(t)= & -\sum_{r} \frac{1}{2}\left(\mathbf{N}_{j}^{r, \omega-} p_{j}^{r,-}+\mathbf{N}_{j}^{r, \omega+} p_{j}^{r,+}\right) \\
& -\sum_{r+1 / 2} \frac{1}{2}\left(\mathbf{N}_{j}^{r+1 / 2, \omega-} p_{j}^{r+1 / 2,-}+\mathbf{N}_{j}^{r+1 / 2, \omega+} p_{j}^{r+1 / 2,+}\right) \\
M_{j} E_{j}^{\prime}(t)= & -\sum_{r} \frac{1}{2}\left(\left(\mathbf{N}_{j}^{r, \omega-} \cdot \mathbf{U}_{r}\right) p_{j}^{r,-}+\left(\mathbf{N}_{j}^{r, \omega+} \cdot \mathbf{U}_{r}\right) p_{j}^{r,+}\right) \\
& -\sum_{r+1 / 2} \frac{1}{2}\left(\left(\mathbf{N}_{j}^{r+1 / 2, \omega-} \cdot \mathbf{U}_{r+1 / 2}\right) p_{j}^{r+1 / 2,-}+\left(\mathbf{N}_{j}^{r+1 / 2, \omega+} \cdot \mathbf{U}_{r+1 / 2}\right) p_{j}^{r+1 / 2,+}\right) .
\end{aligned}\right.
$$

In order to compute $\mathbf{U}_{r}$ and $\mathbf{U}_{r+1 / 2}$ and corresponding pressure $p_{j}^{r, \pm}$ and $p_{j}^{r+1 / 2, \pm}$, we apply the same techniques as in [EUCCLHYD] for nodes and control points. The acoustic Riemann invariant is discretized: (1) for nodes $r$, onto $\mathbf{N}_{j}^{r, \omega \pm}$ directions:

$$
\left\{\begin{array}{c}
p_{j}^{r,+}-p_{j}+\alpha_{j}^{r}\left(\mathbf{U}_{r}-\mathbf{U}_{j}, \hat{\mathbf{N}}_{j}^{r, \omega+}\right)=0 \\
p_{j}^{r,-}-p_{j}+\alpha_{j}^{r}\left(\mathbf{U}_{r}-\mathbf{U}_{j}, \hat{\mathbf{N}}_{j}^{r, \omega-}\right)=0 \\
144
\end{array}\right.
$$




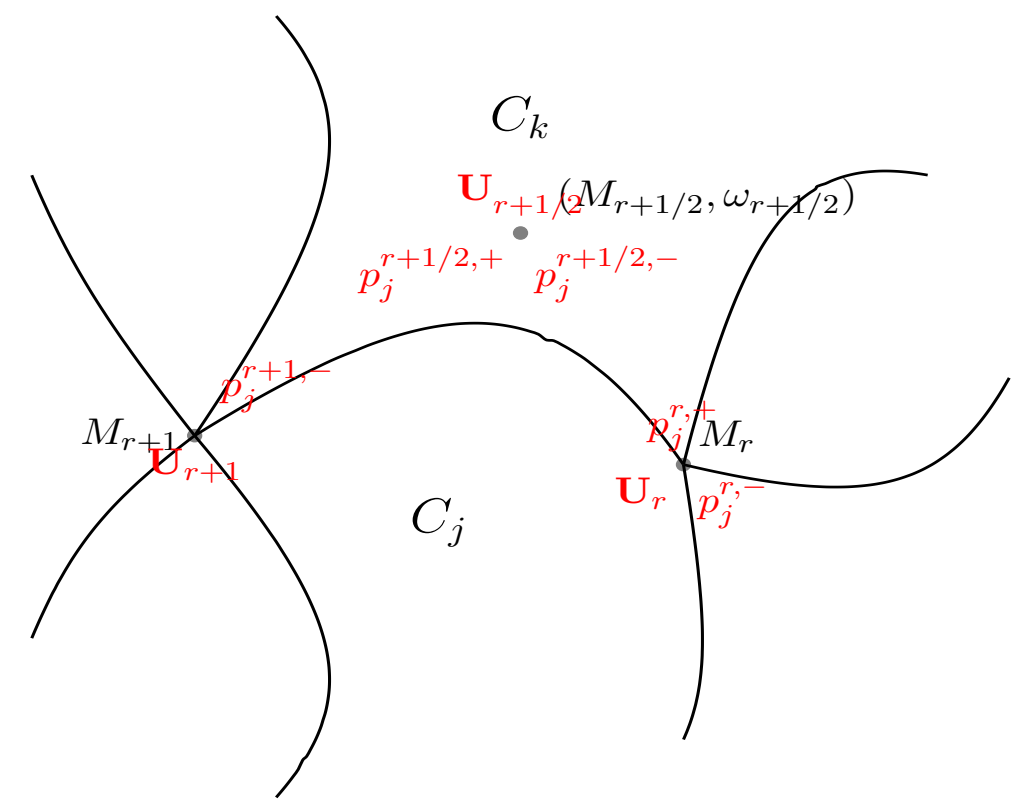

FiguRE 11. Degrees of freedom for Lagrangian $\omega$-EUCCLHYD, intermediate pressure $p_{j}^{d o f, \pm}$ are attached to each effective normal $\mathbf{N}_{j}^{d o f, \omega \pm}$ of Figure (10).

where $\hat{\mathbf{N}}_{j}^{r, \omega \pm}=\frac{\mathbf{N}_{j}^{r, \omega \pm}}{\left|\mathbf{N}_{j}^{r, \omega \pm}\right|}$.

(2) for control points $r+1 / 2$ of edges, onto $\mathbf{N}_{j}^{r+1 / 2, \omega \pm}$ directions:

$$
\left\{\begin{array}{l}
p_{j}^{r+1 / 2,+}-p_{j}+\alpha_{j}^{r+1 / 2}\left(\mathbf{U}_{r+1 / 2}-\mathbf{U}_{j}, \hat{\mathbf{N}}_{j}^{r+1 / 2, \omega+}\right)=0, \\
p_{j}^{r+1 / 2,-}-p_{j}+\alpha_{j}^{r+1 / 2}\left(\mathbf{U}_{r+1 / 2}-\mathbf{U}_{j}, \hat{\mathbf{N}}_{j}^{r+1 / 2, \omega-}\right)=0,
\end{array}\right.
$$

where $\hat{\mathbf{N}}_{j}^{r+1 / 2, \omega \pm}=\frac{\mathbf{N}_{j}^{r+1 / 2, \omega \pm}}{\left|\mathbf{N}_{j}^{r+1 / 2, \omega \pm}\right|}$.

Here $\alpha_{j}^{r}$ and $\alpha_{j}^{r+1 / 2}$ in (34), (35) are the acoustic impedance $\rho_{j} c_{j}\left(c\right.$ is the sound speed $c^{2}=\left.\frac{\partial P}{\partial \rho}\right|_{S}$, and $\mathrm{S}$ the entropy) of the cell $C_{j}$. To construct the solver we also enforce the following conservation conditions:

- Around internal nodes:

$$
\sum_{j} p_{j}^{r,+} \mathbf{N}_{j}^{r, \omega+}+p_{j}^{r,-} \mathbf{N}_{j}^{r, \omega-}=\mathbf{0}
$$

- Around control point of internal edges:

$$
\begin{aligned}
& \sum_{j} p_{j}^{r+1 / 2,+} \mathbf{N}_{j}^{r+1 / 2, \omega+}+p_{j}^{r+1 / 2,-} \mathbf{N}_{j}^{r+1 / 2, \omega-}=\mathbf{0}, \quad \text { which is equivalent to } \\
& p_{j}^{r+1 / 2,+} \mathbf{N}_{j}^{r+1 / 2, \omega+}+p_{j}^{r+1 / 2,-} \mathbf{N}_{j}^{r+1 / 2, \omega-}+p_{k}^{r+1 / 2,+} \mathbf{N}_{k}^{r+1 / 2, \omega+}+p_{k}^{r+1 / 2,-} \mathbf{N}_{k}^{r+1 / 2, \omega-}=\mathbf{0 .}
\end{aligned}
$$

In this case, thanks to the relations $\mathbf{N}_{j}^{r+1 / 2, \omega+}=-\mathbf{N}_{k}^{r+1 / 2, \omega-}, \mathbf{N}_{j}^{r+1 / 2, \omega-}=-\mathbf{N}_{k}^{r+1 / 2, \omega+}$ and $\mathbf{N}_{k}^{r+1 / 2, \omega+}+$ $\mathbf{N}_{k}^{r+1 / 2, \omega-}+\mathbf{N}_{j}^{r+1 / 2, \omega+}+\mathbf{N}_{j}^{r+1 / 2, \omega-}=\mathbf{0}$ (see (32)(30)), we obtain:

$$
f\left(\omega_{r+1 / 2}\right)\left(\left(p_{j}^{r+1 / 2,+}-p_{k}^{r+1 / 2,-}\right) \mathbf{N}_{r+1 / 2, r+1}+\left(p_{j}^{r+1 / 2,-}-p_{k}^{r+1 / 2,+}\right) \mathbf{N}_{r, r+1 / 2}\right)=\mathbf{0} .
$$


To solve (34)(36), we consider the following two by two linear systems and obtain the velocities at each node:

$$
\left\{\begin{array}{l}
A_{r} \mathbf{U}_{r}=\mathbf{b}_{r}, \quad \text { with } \\
A_{r}=\sum_{j} \rho_{j} c_{j}\left(\frac{\mathbf{N}_{j}^{r, \omega+} \otimes \mathbf{N}_{j}^{r, \omega+}}{\left|\mathbf{N}_{j}^{r, \omega+}\right|}+\frac{\mathbf{N}_{j}^{r, \omega-} \otimes \mathbf{N}_{j}^{r, \omega-}}{\left|\mathbf{N}_{j}^{r, \omega-}\right|}\right), \\
b_{r}=\sum_{j}\left(\mathbf{N}_{j}^{r, \omega+}+\mathbf{N}_{j}^{r, \omega-}\right) p_{j}+\rho_{j} c_{j}\left(\frac{\mathbf{N}_{j}^{r, \omega+} \otimes \mathbf{N}_{j}^{r, \omega+}}{\left|\mathbf{N}_{j}^{r, \omega+}\right|}+\frac{\mathbf{N}_{j}^{r, \omega-} \otimes \mathbf{N}_{j}^{r, \omega-}}{\left|\mathbf{N}_{j}^{r, \omega-}\right|}\right) \mathbf{U}_{j} .
\end{array}\right.
$$

This system is always invertible whatever the weight value for edges impinging at node $r$.

For control point (35)(38), we solve:

$$
\left\{\begin{array}{l}
\left.A_{r+1 / 2} \mathbf{U}_{r+1 / 2}=\mathbf{b}_{r+1 / 2}, \quad \text { with (Let : } \Gamma_{r+1 / 2}:=\left(\frac{\mathbf{N}_{r+1 / 2, r+1} \otimes \mathbf{N}_{r+1 / 2, r+1}}{\left|\mathbf{N}_{r+1 / 2, r+1}\right|}+\frac{\mathbf{N}_{r, r+1 / 2} \otimes \mathbf{N}_{r, r+1 / 2}}{\left|\mathbf{N}_{r, r+1 / 2}\right|}\right)\right) \\
A_{r+1 / 2}=f\left(\omega_{r+1 / 2}\right)\left(\rho_{j} c_{j}+\rho_{k} c_{k}\right) \Gamma_{r+1 / 2}, \\
b_{r+1 / 2}=f\left(\omega_{r+1 / 2}\right)\left(\left(\mathbf{N}_{r+1 / 2, r+1}+\mathbf{N}_{r, r+1 / 2}\right)\left(p_{j}-p_{k}\right)+\Gamma_{r+1 / 2}\left(\rho_{j} c_{j} \mathbf{U}_{j}+\rho_{k} c_{k} \mathbf{U}_{k}\right)\right) .
\end{array}\right.
$$

In this case, there are two situations depending on $A_{r+1 / 2}$ :

(1) Invertible : After solving the problem for $\mathbf{U}_{r+1 / 2}$ in (40), the pressures $p_{j}^{r+1 / 2, \omega \pm}$ are computed by mean of (35).

(2) Not Invertible: it means that the problem is only one dimensional, and we first apply the one dimensional Riemann solver that gives normal speed (see also [CEMRACS10]), let $\mathbf{n}_{\mathbf{j}}^{\mathbf{r + 1 / 2 , \omega}}=\frac{C_{j}^{r+1 / 2, \omega}}{\left|C_{j}^{r+1 / 2, \omega}\right|}$ (see (31)):

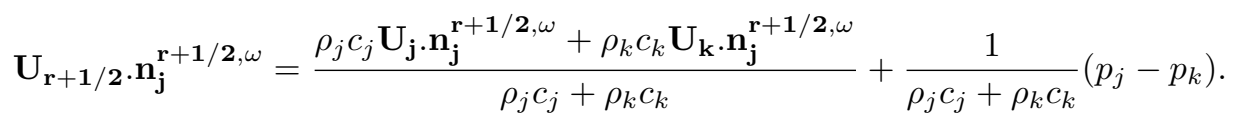

To obtain a truly $2 \mathrm{D}$ speed for the control point, we approximate the tangential part $\mathbf{U}_{\mathbf{r}+\mathbf{1} / \mathbf{2}} \cdot \tau_{\mathbf{j}}^{\mathbf{r + 1 / 2 , \omega}}$ (with $\tau_{\mathbf{j}}^{\mathbf{r}+\mathbf{1} / \mathbf{2}, \omega} \cdot \mathbf{n}_{\mathbf{j}}^{\mathbf{r}+\mathbf{1} / \mathbf{2}, \omega}=\mathbf{0}$ ), see e.g. [GODUNOV] [POSITLA]:

$$
\mathbf{U}_{\mathbf{r}+\mathbf{1} / \mathbf{2}} \cdot \tau_{\mathbf{j}}^{\mathbf{r}+\mathbf{1} / \mathbf{2}, \omega}=\left\{\begin{array}{l}
\mathbf{U}_{\mathbf{j}} \cdot \tau_{\mathbf{j}}^{\mathbf{r}+\mathbf{1} / \mathbf{2}, \omega}, \quad \text { if } \mathbf{U}_{\mathbf{r}+\mathbf{1} / \mathbf{2}} \cdot \mathbf{n}_{\mathbf{j}}^{\mathbf{r}+\mathbf{1} / \mathbf{2}, \omega}>0 \\
\mathbf{U}_{\mathbf{k}} \cdot \tau_{\mathbf{j}}^{\mathbf{r}+\mathbf{1} / \mathbf{2}, \omega}, \quad \text { if } \mathbf{U}_{\mathbf{r}+\mathbf{1} / \mathbf{2}} \cdot \mathbf{n}_{\mathbf{j}}^{\mathbf{r}+\mathbf{1} / \mathbf{2}, \omega}<0 \\
\frac{\rho_{j} c_{j} \mathbf{U}_{\mathbf{j}}+\rho_{k} c_{k} \mathbf{U}_{\mathbf{k}}}{\rho_{j} c_{j}+\rho_{k} c_{k}} \cdot \tau_{\mathbf{j}}^{\mathbf{r}+\mathbf{1} / \mathbf{2}, \omega}, \quad \text { otherwise }
\end{array}\right.
$$

This is also a sufficient condition to obtain Galilean invariance. In each case, once velocity is computed by (39) (resp. (40) (41) (42)), pressure $p_{j}^{r, \pm}$ (resp. $p_{j}^{r+1 / 2, \pm}$ ) in (33) is deduced using (34) (resp. (35)).

Remark 5. As for $\omega$-GLACE scheme, when $\omega \rightarrow 0, \omega$-EUCCLHYD scheme tends to the standard polygonal version [EUCCLHYD].

\subsubsection{Numerical Example: Sedov}

In this case, we solve the Sedov problem. The initial condition is given by $\left(\rho^{0}, P^{0}, \mathbf{U}^{0}\right)=\left(1,10^{-6}, \mathbf{0}\right)$ and $\gamma=1.4$. The pressure in cell $\mathrm{C}$ containing the origin is such that, see [HDRPHMAIRE],

$$
P_{c}=(\gamma-1) \rho_{C} \frac{\varepsilon^{0}}{|C|}
$$

where $\varepsilon^{0}=0.244816$ so that the solution consists of a diverging shock whose front is located at radius $\mathrm{R}=1$ at time $\mathrm{t}=1$. Moreover, the density peak reaches the value 6 .

For conical $\omega$-GLACE/EUCCLHYD simulations, the initial control points are set to the middle of logical edges and all the weights are set to $\omega=1$. As mentioned in [CEMRACS10], the mesh instability of the polygonal GLACE version, see Figure 12 (Left), is corrected by the conical version (Right). For the EUCCLHYD version we do not observe significant difference between polygonal and conical versions on this test case. We note that for $\omega$-GLACE and $\omega$-EUCCLHYD schemes, tangled cells are generally much more numerous than in their polygonal counterpart. Such default can be cured using either ALE methodology on conical cells [CEMRACS10] or an extension of the polygonal stabilization method [DESLABSTAB]. 

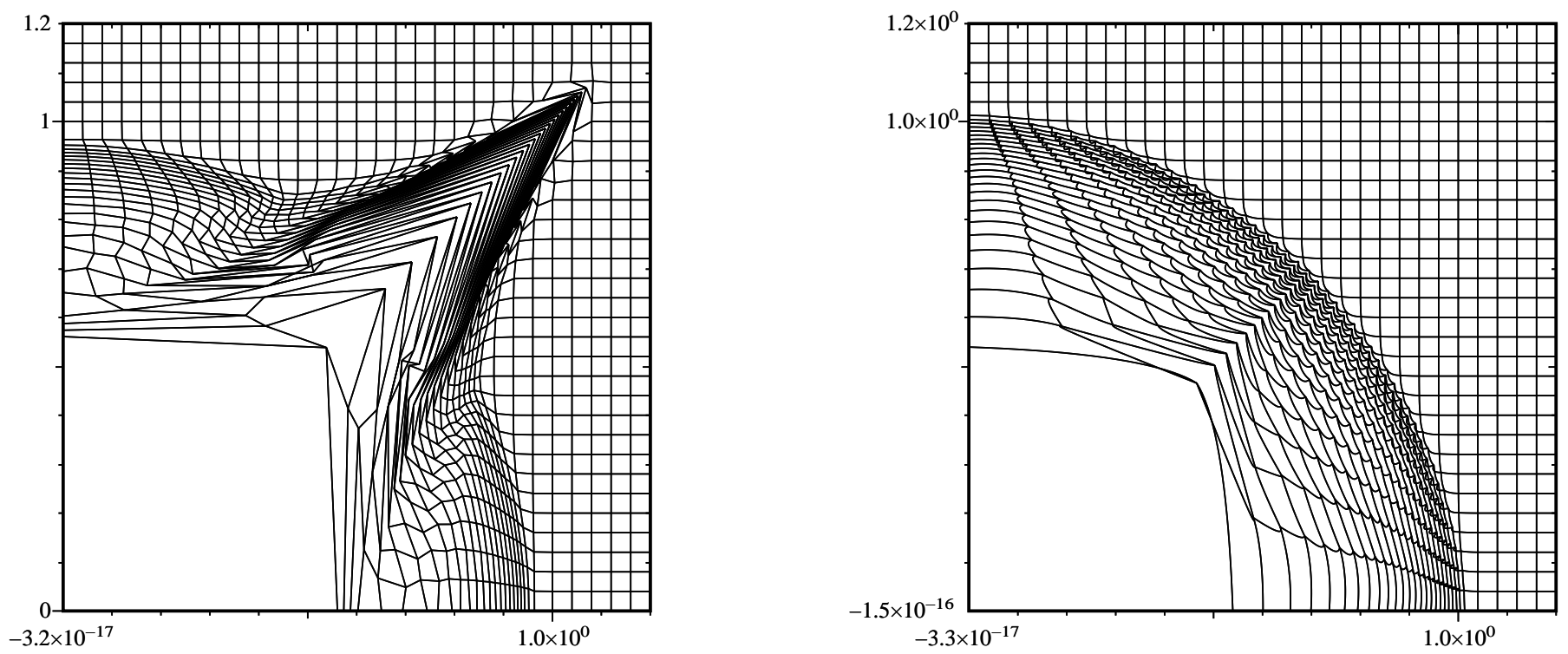

Figure 12. Initial logical mesh made by $30 \times 30$ cells. Meshes at final time $t=1$ for firstorder original polygonal GLACE scheme (left) and conical $\omega$-GLACE scheme (right).
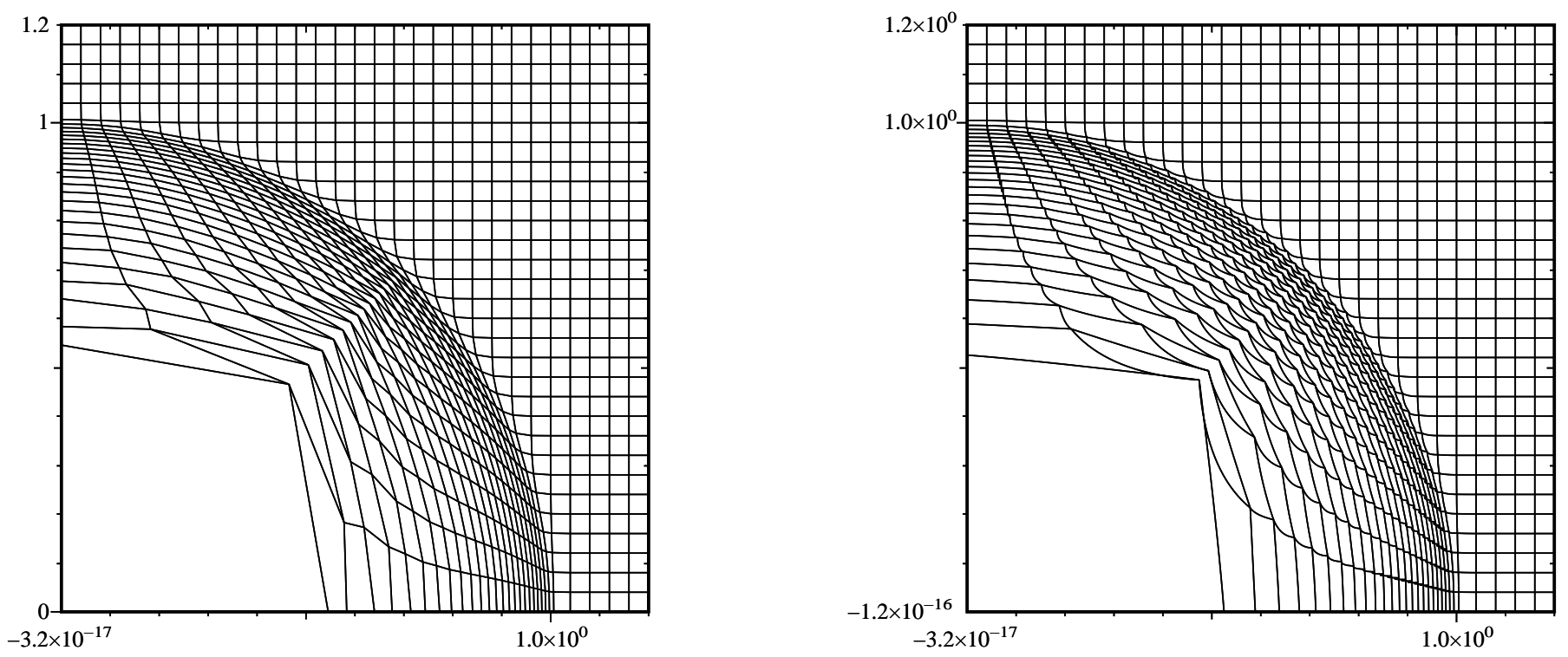

FiguRe 13. Same configuration as Figure (12). Meshes at final time for first-order original polygonal EUCCLHYD scheme (left) and conical $\omega$-EUCCLHYD scheme (right).

\section{2. $(\omega, q)$ - GLACE and EUCCLHYD schemes}

In this second part, we propose to extend GLACE and EUCCLHYD to the case where the degree of freedom is not the control point $M_{r+1 / 2}$ itself but any point on the edge $M^{\omega}\left(q_{r+1 / 2}\right)$ see Figure (15). We 

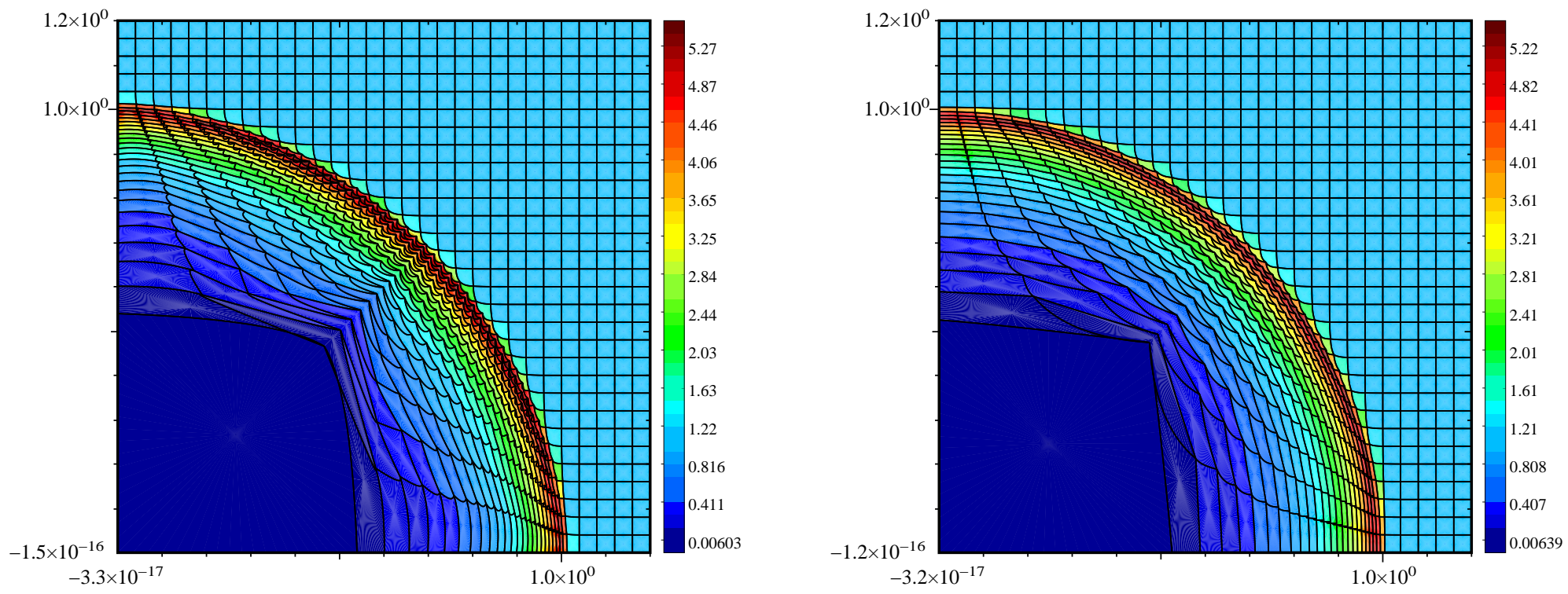

FiguRE 14. The numerical solution (density) of the first-order conical $\omega$-GLACE (Left) and $\omega$-EUCCLHYD (Right) schemes (caution: level of colors are not exactly the same.)

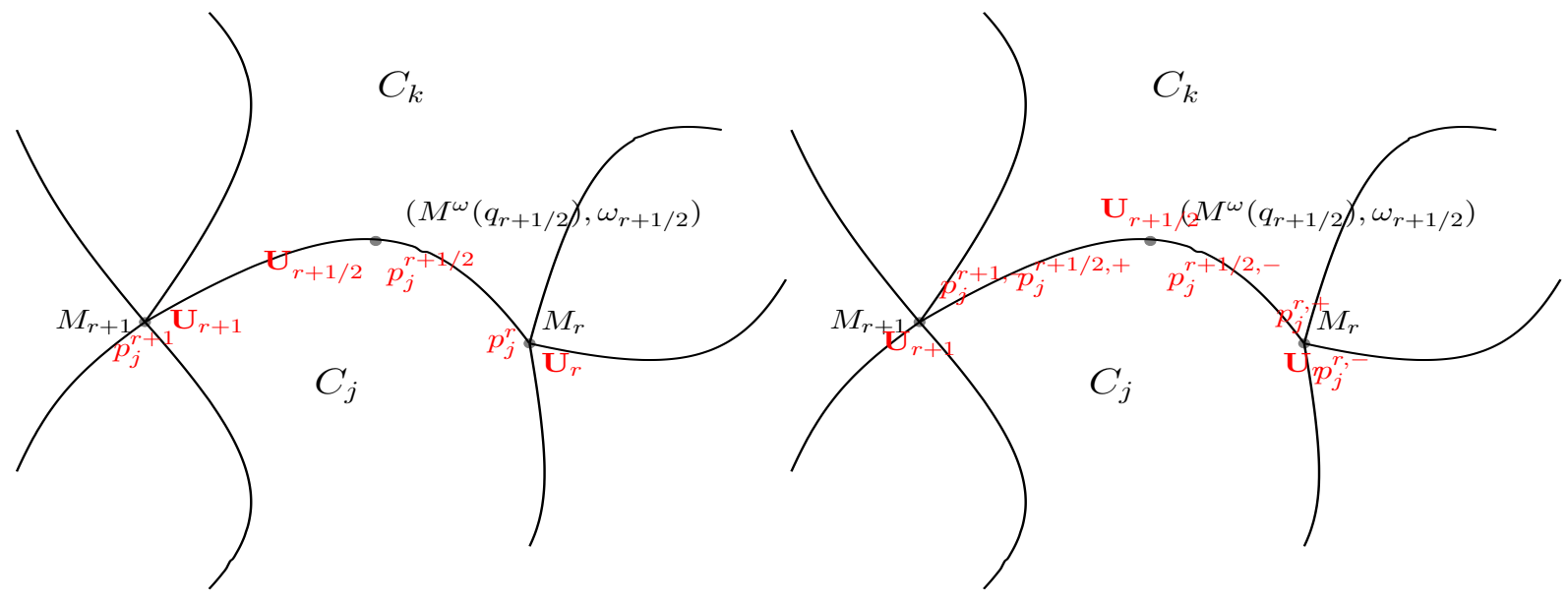

Figure 15. Degrees of freedom for Lagrangian $(\omega, q)$-GLACE (Left) and $(\omega, q)$ EUCCLHYD (Right) scheme on conic cells, the new degrees of freedom are located on the curved edge.

take the notations of (26) (28) (29), see also Figures (7) (Right) and (9) (Right). 
(1) If we denote $M(t)$ the mass of cell $C(t)\left(M(t)=\int_{C(t)} \rho d v\right)$ which is constant, the semi-discrete $(\omega, q)$ GLACE scheme on conic cells writes:

$$
\left\{\begin{array}{l}
M_{j} \tau_{j}^{\prime}(t)=\sum_{r}\left(\tilde{\mathbf{C}}_{j}^{r, \omega}, \mathbf{U}_{r}\right)+\sum_{r+1 / 2}\left(\tilde{\mathbf{C}}_{j}^{r+1 / 2, \omega}, \mathbf{U}_{r+1 / 2}\right), \quad \tau_{j}(t)=\frac{1}{\rho_{j}(t)}, \\
M_{j} \mathbf{U}_{j}^{\prime}(t)=-\sum_{r} \tilde{\mathbf{C}}_{j}^{r, \omega} p_{j}^{r}-\sum_{r+1 / 2} \tilde{\mathbf{C}}_{j}^{r+1 / 2, \omega} p_{j}^{r+1 / 2}, \\
M_{j} E_{j}^{\prime}(t)=-\sum_{r}\left(\tilde{\mathbf{C}}_{j}^{r, \omega}, \mathbf{U}_{r}\right) p_{j}^{r}-\sum_{r+1 / 2}\left(\tilde{\mathbf{C}}_{j}^{r+1 / 2, \omega}, \mathbf{U}_{r+1 / 2}\right) p_{j}^{r+1 / 2},
\end{array}\right.
$$

where the $\tilde{\mathbf{C}}_{j}^{r, \omega}$ and $\tilde{\mathbf{C}}_{j}^{r+1 / 2, \omega}$ are computed in (28) and (29). In order to determine $\mathbf{U}_{r}$ and $\mathbf{U}_{r+1 / 2}$ and the corresponding pressure $p_{j}^{r}$ and $p_{j}^{r+1 / 2}$, we apply the same techniques as in [GLACE] for nodes and $M^{\omega}\left(q_{r+1 / 2}\right)$ points. The Riemann invariant is discretized:

(a) for nodes $r$, onto $\tilde{\mathbf{C}}_{j}^{r, \omega}$ direction:

$$
p_{j}^{r}-p_{j}+\alpha_{j}^{r}\left(\mathbf{U}_{r}-\mathbf{U}_{j}, \tilde{\mathbf{n}}_{j}^{r, \omega}\right)=0
$$

where $\tilde{\mathbf{n}}_{j}^{r, \omega}=\frac{\tilde{\mathbf{C}}_{j}^{r, \omega}}{\left|\tilde{\mathbf{C}}_{j}^{r, \omega}\right|}$ see (28).

(b) for $M^{\omega}\left(q_{r+1 / 2}\right)$ on edges, onto $\tilde{\mathbf{C}}_{j}^{r+1 / 2, \omega}$ direction:

$$
p_{j}^{r+1 / 2}-p_{j}+\alpha_{j}^{r+1 / 2}\left(\mathbf{U}_{r+1 / 2}-\mathbf{U}_{j}, \tilde{\mathbf{n}}_{j}^{r+1 / 2, \omega}\right)=0,
$$

where $\tilde{\mathbf{n}}_{j}^{r+1 / 2, \omega}=\frac{\tilde{\mathbf{C}}_{j}^{r+1 / 2, \omega}}{\left|\tilde{\mathbf{C}}_{j}^{r+1 / 2, \omega}\right|}$ see $(29)$.

Here $\alpha_{j}^{r}$ and $\alpha_{j}^{r+1 / 2}$ in (45), (46) are the acoustic impedance $\rho_{j} c_{j}\left(c\right.$ is the sound speed $c^{2}=\left.\frac{\partial P}{\partial \rho}\right|_{S}$, and $\mathrm{S}$ the entropy) of the cell $C_{j}$. To construct the solver we also enforce the following conservation conditions:

- Around internal nodes:

$$
\sum_{j} p_{j}^{r} \tilde{\mathbf{C}}_{j}^{r, \omega}=\mathbf{0}
$$

- Around $M^{\omega}\left(q_{r+1 / 2}\right)$ on internal edges:

$$
\begin{aligned}
& \sum_{j} p_{j}^{r+1 / 2} \tilde{\mathbf{C}}_{j}^{r+1 / 2, \omega}=\mathbf{0}, \quad \text { which is equivalent to } \\
& p_{j}^{r+1 / 2} \tilde{\mathbf{C}}_{j}^{r+1 / 2, \omega}+p_{k}^{r+1 / 2} \tilde{\mathbf{C}}_{k}^{r+1 / 2, \omega}=\mathbf{0} .
\end{aligned}
$$

Hence in this case, thanks to relation $\tilde{\mathbf{C}}_{k}^{r+1 / 2, \omega}+\tilde{\mathbf{C}}_{j}^{r+1 / 2, \omega}=0$ (see (55)), we obtain the continuity of pressure at $M^{\omega}\left(q_{r+1 / 2}\right)$ point:

$$
p_{j}^{r+1 / 2}=p_{k}^{r+1 / 2}
$$

To solve (45)(47), we consider the following two by two linear systems and obtain the velocities at each node:

$$
\left\{\begin{array}{l}
A_{r} \mathbf{U}_{r}=\mathbf{b}_{r}, \quad \text { with } \\
A_{r}=\sum_{j} \rho_{j} c_{j} \frac{\tilde{\mathbf{C}}_{j}^{r, \omega} \otimes \tilde{\mathbf{C}}_{j}^{r, \omega}}{\left|\tilde{\mathbf{C}}_{j}^{r, \omega}\right|} \\
b_{r}=\sum_{j} \tilde{\mathbf{C}}_{j}^{r, \omega} p_{j}+\rho_{j} c_{j} \frac{\tilde{\mathbf{C}}_{j}^{r, \omega} \otimes \tilde{\mathbf{C}}_{j}^{r, \omega}}{\left|\tilde{\mathbf{C}}_{j}^{r, \omega}\right|} \mathbf{U}_{j}
\end{array}\right.
$$

This system is always invertible whatever the weight value for edges impinging at node $r$. Once the speeds are computed by (50) the pressure $p_{j}^{r}$ in (44) are obtained using (45).

For $M^{\omega}\left(q_{r+1 / 2}\right)$ point (46), we first apply the one dimensional Riemann solver that gives both normal 
speed and continuous pressure (see (49)):

$$
\left\{\begin{array}{l}
\mathbf{U}_{\mathbf{r}+\mathbf{1} / \mathbf{2}} \cdot \tilde{\mathbf{n}}_{\mathbf{j}}^{\mathbf{r}+\mathbf{1} / \mathbf{2}, \omega}=\frac{\rho_{j} c_{j} \mathbf{U}_{\mathbf{j}} \cdot \tilde{\mathbf{n}}_{\mathbf{j}}^{\mathbf{r}+\mathbf{1} / \mathbf{2}, \omega}+\rho_{k} c_{k} \mathbf{U}_{\mathbf{k}} \cdot \tilde{\mathbf{n}}_{\mathbf{j}}^{\mathbf{r}+\mathbf{1} / \mathbf{2}, \omega}}{\rho_{j} c_{j}+\rho_{k} c_{k}}+\frac{1}{\rho_{j} c_{j}+\rho_{k} c_{k}}\left(p_{j}-p_{k}\right), \\
p^{r+1 / 2}=\frac{\rho_{j} c_{j} p_{j}+\rho_{k} c_{k} p_{k}}{\rho_{j} c_{j}+\rho_{k} c_{k}}+\frac{\rho_{j} c_{j} \rho_{k} c_{k}}{\rho_{j} c_{j}+\rho_{k} c_{k}}\left(\mathbf{U}_{\mathbf{j}}-\mathbf{U}_{\mathbf{k}}\right) \cdot \tilde{\mathbf{n}}_{\mathbf{j}}^{\mathbf{r}+\mathbf{1} / \mathbf{2}, \omega}
\end{array}\right.
$$

To obtain a truly $2 \mathrm{D}$ speed for the $M^{\omega}\left(q_{r+1 / 2}\right)$ point, we approximate the tangential part $\mathbf{U}_{\mathbf{r}+\mathbf{1} / \mathbf{2}} \cdot \tilde{\tau}_{\mathbf{j}}^{\mathbf{r}+\mathbf{1 / 2}, \omega}$ (with $\tilde{\tau}_{\mathbf{j}}^{\mathbf{r}+\mathbf{1 / 2}, \omega} \cdot \tilde{\mathbf{n}}_{\mathbf{j}}^{\mathbf{r}+\mathbf{1} / \mathbf{2}, \omega}=\mathbf{0}$ ), see e.g. [GODUNOV] [POSITLA]:

$$
\mathbf{U}_{\mathbf{r}+\mathbf{1} / \mathbf{2}} \cdot \tilde{\tau}_{\mathbf{j}}^{\mathbf{r + 1 / 2}, \omega}=\left\{\begin{array}{l}
\mathbf{U}_{\mathbf{j}} \cdot \tilde{\tau}_{\mathbf{j}}^{\mathbf{r}+\mathbf{1} / \mathbf{2}, \omega}, \quad \text { if } \mathbf{U}_{\mathbf{r}+\mathbf{1} / \mathbf{2}} \cdot \tilde{\mathbf{n}}_{\mathbf{j}}^{\mathbf{r + 1 / 2}, \omega}>0, \\
\mathbf{U}_{\mathbf{k}} \cdot \tilde{\tau}_{\mathbf{j}}^{\mathbf{r}+\mathbf{1} / \mathbf{2}, \omega}, \quad \text { if } \mathbf{U}_{\mathbf{r}+\mathbf{1} / \mathbf{2}} \cdot \tilde{\mathbf{n}}_{\mathbf{j}}^{\mathbf{r}+\mathbf{1} / \mathbf{2}, \omega}<0, \\
\frac{\rho_{j} c_{j} \mathbf{U}_{\mathbf{j}}+\rho_{k} c_{k} \mathbf{U}_{\mathbf{k}}}{\rho_{j} c_{j}+\rho_{k} c_{k}}, \tilde{\tau}_{\mathbf{j}}^{\mathbf{r}+\mathbf{1} / \mathbf{2}, \omega}, \quad \text { otherwise. }
\end{array}\right.
$$

(2) $(\omega, q)$-EUCCLHYD scheme. To obtain new EUCCLHYD normals, we define:

$$
\left\{\begin{array}{l}
\tilde{\mathbf{N}}_{j}^{r, \omega-}:=\left(1-g\left(\omega_{r-1 / 2}, q_{r-1 / 2}\right)\right) \mathbf{N}_{r-1, r}+g\left(\omega_{r-1 / 2}, q_{r-1 / 2}\right) \tilde{\mathbf{N}}_{r-1 / 2, r} \\
\tilde{\mathbf{N}}_{j}^{r, \omega+}:=\left(1-g\left(\omega_{r+1 / 2}, q_{r+1 / 2}\right)\right) \mathbf{N}_{r, r+1}+g\left(\omega_{r+1 / 2}, q_{r+1 / 2}\right) \tilde{\mathbf{N}}_{r, r+1 / 2} \\
\tilde{\mathbf{N}}_{j}^{r+1 / 2, \omega-}:=g\left(\omega_{r+1 / 2}, q_{r+1 / 2}\right) \tilde{\mathbf{N}}_{r, r+1 / 2} \\
\tilde{\mathbf{N}}_{j}^{r+1 / 2, \omega+}:=g\left(\omega_{r+1 / 2}, q_{r+1 / 2}\right) \tilde{\mathbf{N}}_{r+1 / 2, r+1}
\end{array}\right.
$$

(a) We have the following link between $\tilde{C}_{j}^{r, \omega}$ "GLACE vectors" (28) (29) see Figure (9) and "EUCCLHYD vectors" defined by (53):

$$
\left\{\begin{array}{l}
\tilde{\mathbf{C}}_{j}^{r, \omega}=\frac{1}{2}\left(\tilde{\mathbf{N}}_{j}^{r, \omega-}+\tilde{\mathbf{N}}_{j}^{r, \omega+}\right) \\
\tilde{\mathbf{C}}_{j}^{r+1 / 2, \omega}=\frac{1}{2}\left(\tilde{\mathbf{N}}_{j}^{r+1 / 2, \omega-}+\tilde{\mathbf{N}}_{j}^{r+1 / 2, \omega+}\right)
\end{array}\right.
$$

(b) Moreover, we have the following identities:

$$
\left\{\begin{array}{l}
\text { For each cell } C_{j}: \sum_{r}\left(\tilde{\mathbf{N}}_{j}^{r, \omega-}+\tilde{\mathbf{N}}_{j}^{r, \omega+}\right)+\sum_{r+1 / 2}\left(\tilde{\mathbf{N}}_{j}^{r+1 / 2, \omega-}+\tilde{\mathbf{N}}_{j}^{r+1 / 2, \omega-}\right)=0, \\
\text { for internal node } M_{r}: \sum_{j}\left(\tilde{\mathbf{N}}_{j}^{r, \omega-}+\tilde{\mathbf{N}}_{j}^{r, \omega+}\right)=0, \\
\text { and two adjacent cells } \mathrm{j} \text { and k : } \tilde{\mathbf{N}}_{j}^{r+1 / 2, \omega-}+\tilde{\mathbf{N}}_{j}^{r+1 / 2, \omega+}+\tilde{\mathbf{N}}_{k}^{r+1 / 2, \omega-}+\tilde{\mathbf{N}}_{k}^{r+1 / 2, \omega+}=0 .
\end{array}\right.
$$

The semi-discrete $(\omega, q)$-EUCCLHYD scheme on conic cells writes:

$$
\left\{\begin{aligned}
M_{j} \tau_{j}^{\prime}(t)= & \sum_{r} \frac{1}{2}\left(\tilde{\mathbf{N}}_{j}^{r, \omega-}+\tilde{\mathbf{N}}_{j}^{r, \omega+}\right) \cdot \mathbf{U}_{r}+\sum_{r+1 / 2} \frac{1}{2}\left(\tilde{\mathbf{N}}_{j}^{r+1 / 2, \omega-}+\tilde{\mathbf{N}}_{j}^{r+1 / 2, \omega+}\right) \cdot \mathbf{U}_{r+1 / 2}, \tau_{j}(t)=\frac{1}{\rho_{j}(t)}, \\
M_{j} \mathbf{U}_{j}^{\prime}(t)= & -\sum_{r} \frac{1}{2}\left(\tilde{\mathbf{N}}_{j}^{r, \omega-} p_{j}^{r,-}+\tilde{\mathbf{N}}_{j}^{r, \omega+} p_{j}^{r,+}\right) \\
& -\sum_{r+1 / 2} \frac{1}{2}\left(\tilde{\mathbf{N}}_{j}^{r+1 / 2, \omega-} p_{j}^{r+1 / 2,-}+\tilde{\mathbf{N}}_{j}^{r+1 / 2, \omega+} p_{j}^{r+1 / 2,+}\right) \\
M_{j} E_{j}^{\prime}(t)= & -\sum_{r} \frac{1}{2}\left(\left(\tilde{\mathbf{N}}_{j}^{r, \omega-} \cdot \mathbf{U}_{r}\right) p_{j}^{r,-}+\left(\tilde{\mathbf{N}}_{j}^{r, \omega+} \cdot \mathbf{U}_{r}\right) p_{j}^{r,+}\right) \\
& -\sum_{r+1 / 2} \frac{1}{2}\left(\left(\tilde{\mathbf{N}}_{j}^{r+1 / 2, \omega-} \cdot \mathbf{U}_{r+1 / 2}\right) p_{j}^{r+1 / 2,-}+\left(\tilde{\mathbf{N}}_{j}^{r+1 / 2, \omega+} \cdot \mathbf{U}_{r+1 / 2}\right) p_{j}^{r+1 / 2,+}\right) .
\end{aligned}\right.
$$

In order to determine $\mathbf{U}_{r}$ and $\mathbf{U}_{r+1 / 2}$ and the corresponding pressure $p_{j}^{r, \pm}$ and $p_{j}^{r+1 / 2, \pm}$, we apply the same techniques as in [EUCCLHYD] for nodes and $M^{\omega}\left(q_{r+1 / 2}\right)$ points. The Riemann invariant is discretized:

(a) for nodes $r$, onto $\tilde{\mathbf{N}}_{j}^{r, \omega \pm}$ directions:

$$
\left\{\begin{array}{c}
p_{j}^{r,+}-p_{j}+\alpha_{j}^{r}\left(\mathbf{U}_{r}-\mathbf{U}_{j}, \hat{\mathbf{N}}_{j}^{r, \omega+}\right)=0 \\
p_{j}^{r,-}-p_{j}+\alpha_{j}^{r}\left(\mathbf{U}_{r}-\mathbf{U}_{j}, \hat{\mathbf{N}}_{j}^{r, \omega-}\right)=0,
\end{array}\right.
$$

where $\hat{\mathbf{N}}_{j}^{r, \omega \pm}=\frac{\tilde{\mathbf{N}}_{j}^{r, \omega \pm}}{\left|\tilde{\mathbf{N}}_{j}^{r, \omega \pm}\right|}$. 
(b) for $M^{\omega}\left(q_{r+1 / 2}\right)$ on edges, onto $\tilde{\mathbf{N}}_{j}^{r+1 / 2, \omega \pm}$ directions:

$$
\left\{\begin{array}{l}
p_{j}^{r+1 / 2,+}-p_{j}+\alpha_{j}^{r+1 / 2}\left(\mathbf{U}_{r+1 / 2}-\mathbf{U}_{j}, \hat{\mathbf{N}}_{j}^{r+1 / 2, \omega+}\right)=0 \\
p_{j}^{r+1 / 2,-}-p_{j}+\alpha_{j}^{r+1 / 2}\left(\mathbf{U}_{r+1 / 2}-\mathbf{U}_{j}, \hat{\mathbf{N}}_{j}^{r+1 / 2, \omega-}\right)=0
\end{array}\right.
$$

where $\hat{\mathbf{N}}_{j}^{r+1 / 2, \omega \pm}=\frac{\tilde{\mathbf{N}}_{j}^{r+1 / 2, \omega \pm}}{\left|\tilde{\mathbf{N}}_{j}^{r+1 / 2, \omega \pm}\right|}$.

Here $\alpha_{j}^{r}$ and $\alpha_{j}^{r+1 / 2}$ in (57), (58) are the acoustic impedance $\rho_{j} c_{j}\left(c\right.$ is the sound speed $c^{2}=\left.\frac{\partial P}{\partial \rho}\right|_{S}$, and $\mathrm{S}$ the entropy) of the cell $C_{j}$. To construct the solver we also enforce the following conservation conditions:

- Around internal nodes:

$$
\sum_{j} p_{j}^{r,+} \tilde{\mathbf{N}}_{j}^{r, \omega+}+p_{j}^{r,-} \tilde{\mathbf{N}}_{j}^{r, \omega-}=\mathbf{0}
$$

- Around $M^{\omega}\left(q_{r+1 / 2}\right)$ on internal edges:

$$
\begin{aligned}
& \sum_{j} p_{j}^{r+1 / 2,+} \tilde{\mathbf{N}}_{j}^{r+1 / 2, \omega+}+p_{j}^{r+1 / 2,-} \tilde{\mathbf{N}}_{j}^{r+1 / 2, \omega-}=\mathbf{0}, \quad \text { which is equivalent to } \\
& p_{j}^{r+1 / 2,+} \tilde{\mathbf{N}}_{j}^{r+1 / 2, \omega+}+p_{j}^{r+1 / 2,-} \tilde{\mathbf{N}}_{j}^{r+1 / 2, \omega-}+p_{k}^{r+1 / 2,+} \tilde{\mathbf{N}}_{k}^{r+1 / 2, \omega+}+p_{k}^{r+1 / 2,-} \tilde{\mathbf{N}}_{k}^{r+1 / 2, \omega-}=\mathbf{0 .}
\end{aligned}
$$

In this case, thanks to the relations $\tilde{\mathbf{N}}_{j}^{r+1 / 2, \omega+}=-\tilde{\mathbf{N}}_{k}^{r+1 / 2, \omega-}, \tilde{\mathbf{N}}_{j}^{r+1 / 2, \omega-}=-\tilde{\mathbf{N}}_{k}^{r+1 / 2, \omega+}$ and $\tilde{\mathbf{N}}_{k}^{r+1 / 2, \omega+}+\tilde{\mathbf{N}}_{k}^{r+1 / 2, \omega-}+\tilde{\mathbf{N}}_{j}^{r+1 / 2, \omega+}+\tilde{\mathbf{N}}_{j}^{r+1 / 2, \omega-}=0$ see (55)(53), we obtain:

$$
f\left(\omega_{r+1 / 2}\right)\left(\left(p_{j}^{r+1 / 2,+}-p_{k}^{r+1 / 2,-}\right) \tilde{\mathbf{N}}_{r+1 / 2, r+1}+\left(p_{j}^{r+1 / 2,-}-p_{k}^{r+1 / 2,+}\right) \tilde{\mathbf{N}}_{r, r+1 / 2}\right)=0 .
$$

To solve (57)(59), we consider the following two by two linear systems and obtain the velocities at each node:

$$
\left\{\begin{array}{l}
A_{r} \mathbf{U}_{r}=\mathbf{b}_{r}, \quad \text { with } \\
A_{r}=\sum_{j} \rho_{j} c_{j}\left(\frac{\tilde{\mathbf{N}}_{j}^{r, \omega+} \otimes \tilde{\mathbf{N}}_{j}^{r, \omega+}}{\left|\tilde{\mathbf{N}}_{j}^{r, \omega+}\right|}+\frac{\tilde{\mathbf{N}}_{j}^{r, \omega-} \otimes \tilde{\mathbf{N}}_{j}^{r, \omega-}}{\left|\tilde{\mathbf{N}}_{j}^{r, \omega-}\right|}\right), \\
b_{r}=\sum_{j}\left(\tilde{\mathbf{N}}_{j}^{r, \omega+}+\tilde{\mathbf{N}}_{j}^{r, \omega-}\right) p_{j}+\rho_{j} c_{j}\left(\frac{\tilde{\mathbf{N}}_{j}^{r, \omega+} \otimes \tilde{\mathbf{N}}_{j}^{r, \omega+}}{\left|\tilde{\mathbf{N}}_{j}^{r, \omega+}\right|}+\frac{\tilde{\mathbf{N}}_{j}^{r, \omega-} \otimes \tilde{\mathbf{N}}_{j}^{r, \omega-}}{\left|\tilde{\mathbf{N}}_{j}^{r, \omega-}\right|}\right) \mathbf{U}_{j} .
\end{array}\right.
$$

This system is always invertible whatever the weight value for edges impinging at node $r$.

For $M^{\omega}\left(q_{r+1 / 2}\right)$ point (58)(61), we solve:

$$
\left\{\begin{array}{l}
A_{r+1 / 2} \mathbf{U}_{r+1 / 2}=\mathbf{b}_{r+1 / 2}, \quad \text { with }\left(\text { Let }: \tilde{\Gamma}_{r+1 / 2}:=\left(\frac{\tilde{\mathbf{N}}_{r+1 / 2, r+1} \otimes \tilde{\mathbf{N}}_{r+1 / 2, r+1}}{\left|\tilde{\mathbf{N}}_{r+1 / 2, r+1}\right|}+\frac{\tilde{\mathbf{N}}_{r, r+1 / 2} \otimes \tilde{\mathbf{N}}_{r, r+1 / 2}}{\left|\tilde{\mathbf{N}}_{r, r+1 / 2}\right|}\right)\right) \\
A_{r+1 / 2}=f\left(\omega_{r+1 / 2}\right)\left(\rho_{j} c_{j}+\rho_{k} c_{k}\right) \tilde{\Gamma}_{r+1 / 2}, \\
b_{r+1 / 2}=f\left(\omega_{r+1 / 2}\right)\left(\left(\tilde{\mathbf{N}}_{r+1 / 2, r+1}+\tilde{\mathbf{N}}_{r, r+1 / 2}\right)\left(p_{j}-p_{k}\right)+\tilde{\Gamma}_{r+1 / 2}\left(\rho_{j} c_{j} \mathbf{U}_{j}+\rho_{k} c_{k} \mathbf{U}_{k}\right)\right) .
\end{array}\right.
$$

In this case, there are two situations depending on $A_{r+1 / 2}$ :

(a) Invertible : After solving the problem for $\mathbf{U}_{r+1 / 2}$ in (63), the pressure $p_{j}^{r+1 / 2, \omega \pm}$ are computed by mean of (58).

(b) Not Invertible: it means that the problem is only one dimensional, and we first apply the one dimensional Riemann solver that gives normal speed (see also [CEMRACS10]), let $\tilde{\mathbf{n}}_{\mathbf{j}}^{\mathbf{r}+\mathbf{1 / 2}, \omega}=$ $\frac{\tilde{C}_{j}^{r+1 / 2, \omega}}{\left|\tilde{C}_{j}^{r+1 / 2, \omega}\right|}($ see $(54))$ :

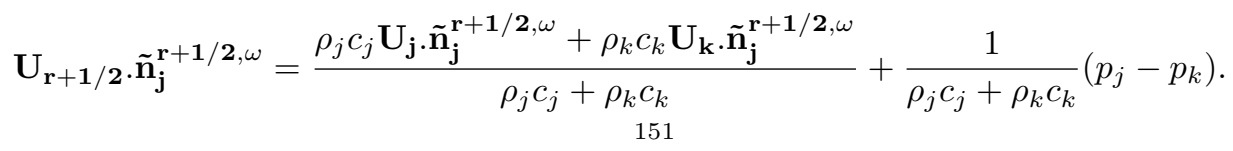


To obtain a truly 2D speed for the $M^{\omega}\left(q_{r+1 / 2}\right)$ point, we approximate the tangential part $\mathbf{U}_{\mathbf{r}+\mathbf{1} / \mathbf{2}} \cdot \tilde{\tau}_{\mathbf{j}}^{\mathbf{r}+\mathbf{1} / \mathbf{2}, \omega}\left(\right.$ with $\left.\tilde{\tau}_{\mathbf{j}}^{\mathbf{r}+\mathbf{1} / \mathbf{2}, \omega} \cdot \tilde{\mathbf{n}}_{\mathbf{j}}^{\mathbf{r}+\mathbf{1} / \mathbf{2}, \omega}=\mathbf{0}\right)$, see e.g. [GODUNOV] [POSITLA]:

$$
\mathbf{U}_{\mathbf{r}+\mathbf{1} / \mathbf{2}} \cdot \tilde{\tau}_{\mathbf{j}}^{\mathbf{r}+\mathbf{1} / \mathbf{2}, \omega}=\left\{\begin{array}{l}
\mathbf{U}_{\mathbf{j}} \cdot \tilde{\tau}_{\mathbf{j}}^{\mathbf{r}+\mathbf{1} / \mathbf{2}, \omega}, \quad \text { if } \mathbf{U}_{\mathbf{r}+\mathbf{1} / \mathbf{2} \cdot \tilde{\mathbf{n}}_{\mathbf{j}}^{\mathbf{r}+\mathbf{1}, \omega}>0} \\
\mathbf{U}_{\mathbf{k}} \cdot \tilde{\tau}_{\mathbf{j}}^{\mathbf{r}+\mathbf{1} / \mathbf{2}, \omega}, \quad \text { if } \mathbf{U}_{\mathbf{r}+\mathbf{1} / \mathbf{2}} \cdot \tilde{\mathbf{n}}_{\mathbf{j}}^{\mathbf{r}+\mathbf{1} / \mathbf{2}, \omega}<0 \\
\frac{\rho_{j} c_{j} \mathbf{U}_{\mathbf{j}}+\rho_{k} c_{k} \mathbf{U}_{\mathbf{k}}}{\rho_{j} c_{j}+\rho_{k} c_{k}} \cdot \tilde{\tau}_{\mathbf{j}}^{\mathbf{r}+\mathbf{1} / \mathbf{2}, \omega}, \quad \text { otherwise }
\end{array}\right.
$$

In each case, once the speeds are computed by (62) (resp. (63) (64) (65)) the pressure $p_{j}^{r, \pm}$ (resp. $p_{j}^{r+1 / 2, \pm}$ ) in (56) are obtained using (57) (resp. (58)).

\section{Continuous in time GCL under Deformation speed}

In our case, we want to extend $\omega$-GLACE or $\omega$-EUCCLHYD formalism, to deforming conical edges, this means that $\omega$ parameter in (5) is now an nonstationary unknown. The cell area is now a function of time dependent weights $\omega_{r+1 / 2}(t)$ :

$$
\left|C_{j}\right|(t)=\frac{1}{2}\left(\sum_{r} \mathbf{C}_{j}^{r, \omega(t)} \cdot \mathbf{O M}_{r}(t)+\sum_{r+1 / 2} \mathbf{C}_{j}^{r+1 / 2, \omega(t)} \cdot \mathbf{O M}_{r+1 / 2}(t)\right):=\frac{1}{2} \sum_{\mathrm{dof}} \mathbf{C}_{j}^{\mathrm{dof}, \omega(t)} \cdot \mathbf{O M}_{\mathrm{dof}}(t) .
$$

The associate time derivative of cell area writes (GCL equation):

$$
\frac{d}{d t}\left|C_{j}(t)\right|=\sum_{r} \mathbf{C}_{j}^{r, \omega(t)} \cdot \mathbf{U}_{r}(t)+\sum_{r+1 / 2} \mathbf{C}_{j}^{r+1 / 2, \omega(t)} \cdot \mathbf{U}_{r+1 / 2}^{t o t}(t)
$$

where

$$
\mathbf{U}_{r+1 / 2}^{t o t}(t):=\mathbf{U}_{r+1 / 2}^{d i s p}(t)+\mathbf{U}_{r+1 / 2}^{\text {defo }}(t),
$$

the total speed acting on each edge is supposed to be an additive decomposition of displacement field and a deformation field.

In the following, we want to recover (67) from (66). First, we have:

$$
\begin{array}{r}
\mathbf{C}_{j}^{r, \omega(t)}=\frac{1}{2}\left(\left(\begin{array}{l}
\left.1-f\left(\omega_{r-1 / 2}(t)\right)\right) \mathbf{N}_{r-1, r}(t)+\left(1-f\left(\omega_{r+1 / 2}(t)\right)\right) \mathbf{N}_{r, r+1}(t) \\
\left.+f\left(\omega_{r-1 / 2}(t)\right) \mathbf{N}_{r-1 / 2, r}(t)+f\left(\omega_{r+1 / 2}(t)\right) \mathbf{N}_{r, r+1 / 2}(t)\right),
\end{array}\right.\right. \\
\mathbf{C}_{j}^{r+1 / 2, \omega(t)}=\frac{f\left(\omega_{r+1 / 2}(t)\right)}{2}\left(\mathbf{N}_{r, r+1 / 2}(t)+\mathbf{N}_{r+1 / 2, r+1}(t)\right) .
\end{array}
$$

These two previous quantities are both vectorial function $\mathbf{g}\left(M_{1}(t), . ., M_{R}(t), M_{1 / 2}(t), . ., M_{R+1 / 2}(t), \omega_{1 / 2}(t), . ., \omega_{R+1 / 2}(t)\right)$ of time dependent variable and then:

$$
\begin{array}{r}
\frac{d}{d t} \mathbf{g}\left(M_{1}(t), . ., M_{R}(t), M_{1 / 2}(t), . ., M_{R+1 / 2}(t), \omega_{1 / 2}(t), . ., \omega_{R+1 / 2}(t)\right)=\sum_{s=1}^{R} \nabla_{M_{s}} \mathbf{g} \cdot \mathbf{O} \dot{M}_{s}(t) \\
+\sum_{s+1 / 2=3 / 2}^{R+1 / 2} \nabla_{M_{s+1 / 2}} \mathbf{g} \cdot \mathbf{O} \dot{M}_{s+1 / 2}(t)+\frac{\partial \mathbf{g}}{\partial \omega_{s+1 / 2}(t)} \dot{\omega}_{s+1 / 2}(t)
\end{array}
$$


we can express:

$$
\begin{aligned}
& \frac{d}{d t}\left|C_{j}(t)\right|=\frac{1}{2}[\underbrace{\sum_{r=1}^{R}\left(\mathbf{C}_{j}^{r, \omega(t)} \cdot \mathbf{O} \mathbf{M}_{r}(t)+\frac{d}{d t} \mathbf{C}_{j}^{r, \omega(t)} \cdot \mathbf{O} \mathbf{M}_{r}(t)\right)}_{A_{R}} \\
& +\underbrace{\sum_{r+1 / 2=3 / 2}^{R+1 / 2}\left(\mathbf{C}_{j}^{r+1 / 2, \omega(t)} \cdot \mathbf{O} \mathbf{M}_{r+1 / 2}(t)+\frac{d}{d t} \mathbf{C}_{j}^{r+1 / 2, \omega(t)} \cdot \mathbf{O} \mathbf{M}_{r+1 / 2}(t)\right)}_{A_{E}}] .
\end{aligned}
$$

Here, we have split the sums (71) in two parts: nodal contributions $A_{R}$ and edges contributions $A_{E}$, we notice that each $\mathbf{C}_{j}^{r, \omega(t)}, \mathbf{C}_{j}^{r+1 / 2, \omega(t)}$ in (69)(70) verifies:

$$
\left\{\begin{aligned}
\frac{d}{d t} C_{j}^{r, \omega(t)}=\sum_{s=1}^{R}\left(\nabla_{M_{s}} C_{j}^{r, \omega(t)} \cdot \mathbf{O M}_{s}(t)+\sum_{s+1 / 2=3 / 2}^{R+1 / 2} \nabla_{M_{s+1 / 2}} C_{j}^{r, \omega(t)} \cdot \mathbf{O} \dot{M}_{s+1 / 2}\right. \\
\left.+\frac{\partial C_{j}^{r, \omega(t)}}{\partial \omega_{s+1 / 2}(t)} \dot{\omega}_{s+1 / 2}(t)\right) \\
\quad \sum_{s=1}^{R}\left(\nabla_{M_{s}} C_{j}^{r+1 / 2, \omega(t)} \cdot \mathbf{O M}_{s}(t)+\sum_{s+1 / 2=3 / 2}^{R+1 / 2} \nabla_{M_{s+1 / 2}} C_{j}^{r+1 / 2, \omega(t)} \cdot \mathbf{O} \dot{M}_{s+1 / 2}\right. \\
\left.\quad+\frac{\partial C_{j}^{r+1 / 2, \omega(t)}}{\partial \omega_{s+1 / 2}(t)} \dot{\omega}_{s+1 / 2}(t)\right) .
\end{aligned}\right.
$$

$$
\begin{aligned}
& A_{R}=\sum_{r=1}^{R} \mathbf{C}_{j}^{r, \omega(t)} \cdot \mathbf{O} \dot{M}_{r}(t) \\
& +\left(\sum_{s=1}^{R} \nabla_{M_{s}} C_{j}^{r, \omega(t)} \cdot \mathbf{O} \dot{M}_{s}(t)+\sum_{s+1 / 2=3 / 2}^{R+1 / 2} \nabla_{M_{s+1 / 2}} C_{j}^{r, \omega(t)} \cdot \mathbf{O} \dot{M}_{s+1 / 2}+\frac{\partial C_{j}^{r, \omega(t)}}{\partial \omega_{s+1 / 2}(t)} \dot{\omega}_{s+1 / 2}(t)\right) \cdot \mathbf{O M} \mathbf{M}_{r}(t) .
\end{aligned}
$$

$$
\begin{aligned}
& A_{E}=\sum_{r+1 / 2=3 / 2}^{R+1 / 2} \mathbf{C}_{j}^{r+1 / 2, \omega(t)} \cdot \mathbf{O} \dot{M}_{r+1 / 2}(t) \\
& +\left(\sum_{s=1}^{R} \nabla_{M_{s}} C_{j}^{r+1 / 2, \omega(t)} \cdot \mathbf{O M}_{s}(t)+\sum_{s+1 / 2=3 / 2}^{R+1 / 2} \nabla_{M_{s+1 / 2}} C_{j}^{r+1 / 2, \omega(t)} \cdot \mathbf{O} \dot{M}_{s+1 / 2}+\frac{\partial C_{j}^{r+1 / 2, \omega(t)}}{\partial \omega_{s+1 / 2}(t)} \dot{\omega}_{s+1 / 2}(t)\right) \cdot \mathbf{O M} \mathbf{M}_{r+1 / 2}(t) .
\end{aligned}
$$

The two last relations (73) (74) rewrite:

$$
\begin{aligned}
& A_{R}=\sum_{r=1}^{R} \mathbf{C}_{j}^{r, \omega(t)} \cdot \mathbf{O M}_{r}(t) \\
& +\sum_{r, s=1}^{R} \underbrace{\left(\mathbf{O M}_{s}(t){ }^{t} \nabla_{M_{s}} C_{j}^{r, \omega(t)} \mathbf{O M}_{r}(t)\right)}_{A_{R}^{1}} \\
& +\sum_{r=1, s+1 / 2=3 / 2}^{R, R+1 / 2} \underbrace{\left(\mathbf{O} \mathbf{M}_{s+1 / 2}(t),{ }^{t} \nabla_{M_{s+1 / 2}} C_{j}^{r, \omega(t)} \mathbf{O M}_{r}(t)\right)}_{A_{R}^{2}}+\underbrace{\dot{\omega}_{s+1 / 2}(t) \frac{\partial C_{j}^{r, \omega(t)}}{\partial \omega_{s+1 / 2}(t)} \cdot \mathbf{O M}_{r}(t)}_{A_{R}^{3}},
\end{aligned}
$$


and for edges:

$$
\begin{aligned}
& A_{E}=\sum_{r+1 / 2=3 / 2}^{R+1 / 2} \mathbf{C}_{j}^{r+1 / 2, \omega(t)} \cdot \mathbf{O M}_{r+1 / 2}(t) \\
& +\sum_{r+1 / 2=3 / 2, s=1}^{R+1 / 2, R} \underbrace{\left(\dot{\mathbf{O M}}_{s}(t),{ }^{t} \nabla_{M_{s}} C_{j}^{r+1 / 2, \omega(t)} \mathbf{O M}_{r+1 / 2}(t)\right)}_{A_{E}^{1}} \\
& +\sum_{(r, s)+1 / 2=3 / 2}^{R+1 / 2} \underbrace{\left(\mathbf{O} \dot{\mathbf{M}}_{s+1 / 2}(t),{ }^{t} \nabla_{M_{s+1 / 2}} C_{j}^{r+1 / 2, \omega(t)} \mathbf{O M}_{r+1 / 2}\right)}_{A_{E}^{2}}+\underbrace{\dot{\omega}_{s+1 / 2}(t) \frac{\partial C_{j}^{r+1 / 2, \omega(t)}}{\partial \omega_{s+1 / 2}(t)} \cdot \mathbf{O M}_{r+1 / 2}(t)}_{A_{E}^{3}} .
\end{aligned}
$$

Using as in [BD], the Maxwell relation on second order derivatives of a potential function: ${ }^{t} \nabla_{M_{s}} C_{j}^{r}=\nabla_{M_{r}} C_{j}^{s}$, we obtain:

$$
\begin{aligned}
A_{R}^{1} & =\sum_{r, s=1}^{R}\left(\mathbf{O} \dot{M}_{s}(t), \nabla_{M_{r}} C_{j}^{s, \omega(t)} \mathbf{O M}_{r}(t)\right), \\
A_{E}^{1} & =\sum_{r+1 / 2, R}^{R+1 / 2=1}\left(\mathbf{O} \dot{M}_{s}(t), \nabla_{M_{r+1 / 2}} C_{j}^{s, \omega(t)} \mathbf{O M}_{r+1 / 2}(t)\right), \\
A_{R}^{2} & =\sum_{r, R+1 / 2=3 / 2}^{R, R+1 / 2}\left(\mathbf{O M}_{s+1 / 2}(t), \nabla_{M_{r}} C_{j}^{s+1 / 2, \omega(t)} \mathbf{O M}_{r}(t)\right), \\
A_{E}^{2} & =\sum_{(r, s)+1 / 2=3 / 2}^{R+1 / 2}\left(\mathbf{O} \mathbf{M}_{s+1 / 2}(t), \nabla_{M_{r+1 / 2}} C_{j}^{s+1 / 2, \omega(t)} \mathbf{O M}_{r+1 / 2}(t)\right), \\
A_{R}^{3} & =\sum_{r, s+1 / 2=3 / 2}^{R, R+1 / 2} \dot{\omega}_{s+1 / 2}(t) \frac{\partial C_{j}^{r, \omega(t)}}{\partial \omega_{s+1 / 2}(t)} \cdot \mathbf{O M}_{r}(t), \\
A_{E}^{3} & =\sum_{(r, s)+1 / 2=3 / 2}^{R+1 / 2} \dot{\omega}_{s+1 / 2}(t) \frac{\partial C_{j}^{r+1 / 2, \omega(t)}}{\partial \omega_{s+1 / 2}(t)} \cdot \mathbf{O M}_{r+1 / 2}(t) .
\end{aligned}
$$

Now, we use the fact that both the $\mathbf{C}_{\mathbf{j}}^{\mathbf{s}, \omega(\mathbf{t})}, \mathbf{C}_{\mathbf{j}}^{\mathbf{s}+\mathbf{1} / \mathbf{2}, \omega(\mathbf{t})}$ are (d-1) homogeneous functions with respect to nodal control point positions:

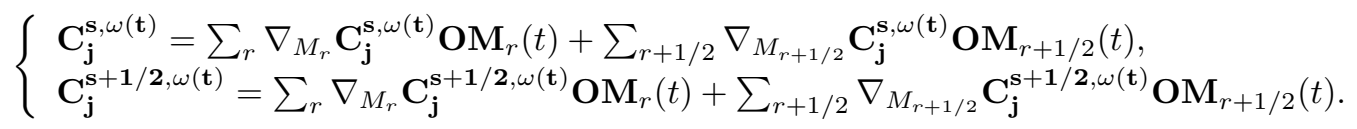

We then get the identities

$$
\begin{aligned}
A_{R}^{1}+A_{E}^{1} & =\sum_{s}\left(\dot{O M}_{s}(t), \mathbf{C}_{\mathbf{j}}^{\mathbf{s}, \omega(\mathbf{t})}\right) \\
A_{R}^{2}+A_{E}^{2} & =\sum_{s+1 / 2}\left(\mathbf{O} \dot{M}_{s+1 / 2}(t), \mathbf{C}_{\mathbf{j}}^{\mathbf{s}+\mathbf{1} / \mathbf{2}, \omega(\mathbf{t})}\right)
\end{aligned}
$$

For the last identity, we need to find an expression for $\nabla_{M_{r}} \frac{\partial\left|C_{j}(t)\right|}{\partial \omega_{s+1 / 2}(t)}$ and $\nabla_{M_{r+1 / 2}} \frac{\partial\left|C_{j}(t)\right|}{\partial \omega_{s+1 / 2}(t)}$ for $A_{3}^{R}, A_{3}^{E}$ in (77). We need first to derive the equation (66) with respect to $\omega_{s+1 / 2}(t)$ :

$$
\frac{\partial\left|C_{j}(t)\right|}{\partial \omega_{s+1 / 2}(t)}=\frac{1}{2}\left(\sum_{r} \frac{\partial C_{j}^{r, \omega(t)}}{\partial \omega_{s+1 / 2}(t)} \cdot \mathbf{O M}_{r}(t)+\sum_{r+1 / 2} \frac{\partial C_{j}^{r+1 / 2, \omega(t)}}{\partial \omega_{s+1 / 2}(t)} \cdot \mathbf{O M}_{r+1 / 2}(t)\right)
$$

This equation (80) is also equal to

$$
\frac{\partial\left|C_{j}(t)\right|}{\partial \omega_{s+1 / 2}(t)}=f^{\prime}\left(\omega_{s+1 / 2}(t)\right) A^{T}\left(M_{s}, M_{s+1 / 2}, M_{s+1}\right) .
$$

To obtain a formalism of GLACE and associate a direction for the deformation speed, we have the relation:

$$
A^{T}\left(M_{s}, M_{s+1 / 2}, M_{s+1}\right)=\frac{1}{f\left(\omega_{s+1 / 2}(t)\right)} \mathbf{C}_{\mathbf{j}}^{\mathbf{s}+\mathbf{1} / \mathbf{2}, \omega(\mathbf{t})} \cdot \mathbf{K M}_{\mathbf{s}+\mathbf{1} / \mathbf{2}}(t) .
$$


Now, since the function (81) is a degree 2 homogeneous function of position:

$$
\frac{\partial\left|C_{j}(t)\right|}{\partial \omega_{s+1 / 2}(t)}=\frac{1}{2}\left(\sum_{r} \nabla_{M_{r}} \frac{\partial\left|C_{j}(t)\right|}{\partial \omega_{s+1 / 2}(t)} \cdot \mathbf{O M}_{r}(t)+\sum_{r+1 / 2} \nabla_{M_{r+1 / 2}} \frac{\partial\left|C_{j}(t)\right|}{\partial \omega_{s+1 / 2}(t)} \cdot \mathbf{O M}_{r+1 / 2}(t)\right)
$$

Then multiplying (83) by $\omega_{s+1 / 2}^{\prime}(t)$ and summing over all edges:

$$
\begin{aligned}
\sum_{s+1 / 2=3 / 2}^{R+1 / 2} \omega_{s+1 / 2}^{\prime}(t) \frac{\partial\left|C_{j}(t)\right|}{\partial \omega_{s+1 / 2}(t)}= & \frac{1}{2}\left(\sum_{r=1, s+1 / 2=3 / 2}^{R, R+1 / 2} \omega_{s+1 / 2}^{\prime}(t) \nabla_{M_{r}} \frac{\partial\left|C_{j}(t)\right|}{\partial \omega_{s+1 / 2}(t)} \cdot \mathbf{O M}_{r}(t)\right. \\
& \left.+\sum_{(r, s)+1 / 2}^{R+1 / 2} \omega_{s+1 / 2}^{\prime}(t) \nabla_{M_{r+1 / 2}} \frac{\partial\left|C_{j}(t)\right|}{\partial \omega_{s+1 / 2}(t)} \cdot \mathbf{O M}_{r+1 / 2}(t)\right) \\
= & \frac{1}{2}\left(A_{R}^{3}+A_{E}^{3}\right) .
\end{aligned}
$$

And we recover these two last terms in (77) and using (82), we obtain:

$$
\frac{1}{2}\left(A_{R}^{3}+A_{E}^{3}\right)=\sum_{s+1 / 2}\left(\frac{f^{\prime}\left(\omega_{s+1 / 2}(t)\right)}{f\left(\omega_{s+1 / 2}(t)\right)} \omega_{s+1 / 2}^{\prime}(t) \mathbf{K M}_{s+1 / 2}(t), \mathbf{C}_{\mathbf{j}}^{\mathbf{s}+\mathbf{1} / \mathbf{2}, \omega(\mathbf{t})}\right) .
$$

Finally, we obtain the evolution of cell area $\frac{d}{d t}\left|C_{j}(t)\right|(71)$ by assembling (79)(84)(75)(76)

$$
\begin{aligned}
\frac{1}{2}\left(A_{R}+A_{E}\right)= & \sum_{s}\left(\mathbf{O} \dot{M}_{s}(t), \mathbf{C}_{\mathbf{j}}^{\mathbf{s}, \omega(\mathbf{t})}\right) \\
& +\sum_{s+1 / 2}\left(\mathbf{O} \dot{M}_{s+1 / 2}(t), \mathbf{C}_{\mathbf{j}}^{\mathbf{s}+\mathbf{1} / \mathbf{2}, \omega(\mathbf{t})}\right)+\left(\frac{f^{\prime}\left(\omega_{s+1 / 2}(t)\right)}{f\left(\omega_{s+1 / 2}(t)\right)} \omega_{s+1 / 2}^{\prime}(t) \mathbf{K M}_{s+1 / 2}(t), \mathbf{C}_{\mathbf{j}}^{\mathbf{s}+\mathbf{1} / \mathbf{2}, \omega(\mathbf{t})}\right),
\end{aligned}
$$

and we recover (67) with:

$$
\left\{\begin{array}{l}
\mathbf{U}_{r}(t)=\mathbf{O M}_{r}(t) \\
\mathbf{U}_{r+1 / 2}^{t o t}(t)=\mathbf{O} \mathbf{M}_{r+1 / 2}(t)+\frac{f^{\prime}\left(\omega_{r+1 / 2}(t)\right)}{f\left(\omega_{r+1 / 2}(t)\right)} \omega_{r+1 / 2}^{\prime}(t) \mathbf{K M}_{r+1 / 2}(t)
\end{array}\right.
$$

Remark 6. - We notice that in (86), the speed associated to the edge deformation in (67)(68), is nothing but:

$$
\mathbf{U}_{r+1 / 2}^{\text {defo }}(t):=\frac{f^{\prime}\left(\omega_{r+1 / 2}(t)\right)}{f\left(\omega_{r+1 / 2}(t)\right)} \omega_{r+1 / 2}^{\prime}(t) \mathbf{K M}_{r+1 / 2}(t)=\frac{d}{d t}\left(\log \left(f\left(\omega_{r+1 / 2}(t)\right)\right)\right) \mathbf{K M}_{r+1 / 2}(t)
$$

- We can recover the same result using the $\tilde{\mathbf{C}}_{\mathbf{j}}^{\mathbf{r}, \omega(\mathbf{t})}$ formalism replacing $\frac{1}{f\left(\omega_{r+1 / 2}(t)\right)} \mathbf{C}_{\mathbf{j}}^{\mathbf{r}+\mathbf{1 / 2}, \omega(\mathbf{t})} \cdot \mathbf{K M}_{\mathbf{r}+\mathbf{1} / \mathbf{2}}$ in (82) by $\frac{1}{g\left(\omega_{r+1 / 2}(t), q_{r+1 / 2}\right)} \tilde{\mathbf{C}}_{\mathbf{j}}^{\mathbf{r}+\mathbf{1} / \mathbf{2}, \omega(\mathbf{t})} \cdot \mathbf{K}\left(\mathbf{q}_{\mathbf{r}+\mathbf{1 / 2}}\right) \mathbf{M}^{\omega}\left(\mathbf{q}_{\mathbf{r}+\mathbf{1 / 2}}\right)$, for any $\left.q_{r+1 / 2} \in\right] 0,1[$ :

$$
\begin{aligned}
\mathbf{U}_{r+1 / 2}^{\text {defo }}(t) & :=\frac{\partial_{\omega} g\left(\omega_{r+1 / 2}(t), q_{r+1 / 2}\right)}{g\left(\omega_{r+1 / 2}(t), q_{r+1 / 2}\right)} \omega_{r+1 / 2}^{\prime}(t) \mathbf{K}\left(\mathbf{q}_{\mathbf{r}+\mathbf{1} / \mathbf{2}}\right) \mathbf{M}^{\omega}\left(\mathbf{q}_{\mathbf{r}+\mathbf{1} / \mathbf{2}}\right)(t) \\
& =\frac{d}{d t}\left(\log \left(g\left(\omega_{r+1 / 2}(t), q_{r+1 / 2}\right)\right)\right) \mathbf{K}\left(\mathbf{q}_{\mathbf{r}+\mathbf{1} / \mathbf{2}}\right) \mathbf{M}^{\omega}\left(\mathbf{q}_{\mathbf{r}+\mathbf{1} / \mathbf{2}}\right)(t), \\
\mathbf{U}_{r+1 / 2}^{\text {disp }}(t) & :=\mathbf{O}^{\omega}\left(\mathbf{q}_{\mathbf{r}+\mathbf{1} / \mathbf{2}}\right)(t) .
\end{aligned}
$$

For any of the two schemes (87) or (88), the only problem is now to compute $\omega^{\prime}(t)$ for arbitrary conical curve. We have some exact geometrical identities for conic curves:

(1) Tangent information (First order): see (6).

(2) Curvature information (Second order):

\section{Proposition 7.}

$$
\frac{d}{d t} \omega_{r+1 / 2}(t)=-4 \omega_{r+1 / 2}^{5}(t) \frac{d}{d t}\left(\frac{\left(\left\|M_{r} M_{r+1 / 2}\right\|\left\|M_{r+1 / 2} M_{r+1}\right\|\right)^{3}}{\left(A^{T}\left(M_{r}, M_{r+1 / 2}, M_{r+1}\right)\right)^{2}} \kappa\left(M_{r}\right) \kappa\left(M_{r+1}\right)\right)
$$

Where $A^{T}\left(M_{r}, M_{r+1 / 2}, M_{r+1}\right)$ is the simplex area of the three points and $\kappa(M)$ the curvature of the conic curve at $M$. 
Proof. For $M=(x(q), y(q))$ belonging to the curve see (5), we use the relation $\kappa(x(q), y(q))=$ $\frac{x^{\prime \prime}(q) y^{\prime}(q)-x^{\prime}(q) y^{\prime \prime}(q)}{\left(x^{\prime}(q)^{2}+y^{\prime}(q)^{2}\right)^{3 / 2}}$, we have the two following relations:

$$
\begin{aligned}
\kappa\left(M_{r}\right) & =\frac{A^{T}\left(M_{r}, M_{r+1 / 2}, M_{r+1}\right)}{\omega^{2}\left\|M_{r} M_{r+1 / 2}\right\|^{3}}, \\
\kappa\left(M_{r+1}\right) & =\frac{A^{T}\left(M_{r}, M_{r+1 / 2}, M_{r+1}\right)}{\omega^{2}\left\|M_{r+1 / 2} M_{r+1}\right\|^{3}},
\end{aligned}
$$

which imply:

$$
\kappa\left(M_{r}\right) \kappa\left(M_{r+1}\right)=\frac{\left(A^{T}\left(M_{r} M_{r+1 / 2} M_{r+1}\right)\right)^{2}}{\omega^{4}|| M_{r} M_{r+1 / 2}\left\|^{3}\right\| M_{r+1 / 2} M_{r+1} \|^{3}} .
$$

\subsection{Particular case: the circle}

We use the $\tilde{\mathbf{C}}_{j}^{r, \omega(t)}$ formalism (see (26)) with $q_{r+1 / 2}=\frac{1}{2}$ for all edges, and we impose the conic for the edge $r+1 / 2$ to be the (unique) circle passing through $M_{r}, M^{\omega}(1 / 2)=S_{r+1 / 2}, M_{r+1}$.

We have

$$
\frac{d}{d t}\left|C_{j}(t)\right|=\sum_{r} \tilde{\mathbf{C}}_{j}^{r, \omega(t)} \cdot \mathbf{U}_{r}(t)+\sum_{r+1 / 2} \tilde{\mathbf{C}}_{j}^{r+1 / 2, \omega(t)} \cdot\left(\mathbf{U}_{r+1 / 2}^{d i s p}(t)+\mathbf{U}_{r+1 / 2}^{d e f o}(t)\right) .
$$

In this particular case, the speed of displacement is given by

$$
\mathbf{U}_{r+1 / 2}^{d i s p}(t)=\frac{1}{2}\left(\mathbf{U}_{r}(t)+\mathbf{U}_{r+1}(t)\right)+\mathbf{l}_{j}^{r+1 / 2 \mathbf{n}_{j}^{r+1 / 2}}
$$

with $\mathbf{K}_{\mathbf{r}+\mathbf{1} / \mathbf{2}} \mathbf{S}_{\mathbf{r}+\mathbf{1} / \mathbf{2}}=l_{j}^{r+1 / 2} \mathbf{n}_{j}^{r+1 / 2}$ and $\mathbf{n}_{j}^{r+1 / 2}=\frac{\tilde{\mathbf{C}}_{j}^{r+1 / 2, \omega(t)}}{\left\|\tilde{\mathbf{C}}_{j}^{r+1 / 2, \omega(t)}\right\|}$

and the speed of deformation is given by

with

$$
\mathbf{U}_{r+1 / 2}^{\text {defo }}(t)=\frac{h^{\prime}\left(\omega_{r+1 / 2}(t)\right)}{h\left(\omega_{r+1 / 2}(t)\right)} \omega_{r+1 / 2}^{\prime}(t) l_{j}^{r+1 / 2}(t) \mathbf{n}_{j}^{r+1 / 2}
$$

$$
h(\omega)=\left(\frac{2}{1-\omega}\right)\left(\frac{1}{\sqrt{1-\omega^{2}}} \operatorname{atan}\left(\sqrt{\frac{1-\omega}{1+\omega}}\right)-\frac{\omega}{2}\right) .
$$

Remark 8. Note that $l_{j}^{r+1 / 2}$ may be positive or negative depending on the sign of $\left(\mathbf{n}_{j}^{r+1 / 2} \cdot \mathbf{K}_{\mathbf{r}+\mathbf{1} / \mathbf{2}} \mathbf{S}_{\mathbf{r}+\mathbf{1 / 2}}\right)$.

Remark 9. We can express $h(\omega)$ in a more compact form as a function of $\theta$ (see Figure 3):

$$
h(\omega)=\frac{\theta-\sin (\theta)}{2 \sin \left(\frac{\theta}{2}\right)-\sin (\theta)} .
$$

We want to express $\mathbf{U}_{r+1 / 2}^{\text {defo }}(t)$ as a function of $\mathbf{U}_{r}(t), \mathbf{U}_{r+1}(t)$ and $\left(l_{j}^{r+1 / 2}\right)^{\prime}(t)$.

$$
\left\{\begin{array}{l}
\omega_{r+1 / 2}=\cos \left(\frac{\theta}{2}\right)=\frac{-\left(l_{j}^{r+1 / 2}\right)^{2}+\frac{d_{r+1 / 2}^{2}}{4}}{\left(l_{j}^{r+1 / 2}\right)^{2}+\frac{d_{r+1 / 2}^{2}}{4}}, \\
d_{r+1 / 2}=\left\|\mathbf{M}_{\mathbf{r}} \mathbf{M}_{\mathbf{r}+\mathbf{1}}\right\| .
\end{array}\right.
$$

We have

$$
\mathbf{U}_{r+1 / 2}^{\text {defo }}(t)=\frac{h^{\prime}\left(\omega_{r+1 / 2}(t)\right)}{h\left(\omega_{r+1 / 2}(t)\right)} \omega_{r+1 / 2}^{\prime}(t) l_{j}^{r+1 / 2}(t) \mathbf{n}_{j}^{r+1 / 2} .
$$


Noting that $\omega_{r+1 / 2}$ in (94) is a function of $M_{r}, M_{r+1}, l_{j}^{r+1 / 2}$, we can express $\omega_{r+1 / 2}^{\prime}(t)$ as a function of $\mathbf{U}_{r}(t)$, $\mathbf{U}_{r+1}(t)$ and $\left(l_{j}^{r+1 / 2}\right)^{\prime}(t)$ :

$$
\omega_{r+1 / 2}^{\prime}(t)=\left(\mathbf{U}_{r}(t), \nabla_{\mathbf{M}_{\mathbf{r}}}\left(\omega_{r+1 / 2}\right)\right)+\left(\mathbf{U}_{r+1}(t), \nabla_{\mathbf{M}_{\mathbf{r}+1}}\left(\omega_{r+1 / 2}\right)\right)+\frac{\partial \omega_{r+1 / 2}}{\partial l}\left(l_{j}^{r+1 / 2}\right)^{\prime}(t) .
$$

We have $\nabla_{\mathbf{M}_{\mathbf{r}}}\left(d_{r+1 / 2}\right)=\mathbf{n}^{r, r+1 / 2}$ and $\nabla_{\mathbf{M}_{\mathbf{r}+1}}\left(d_{r+1 / 2}\right)=\mathbf{n}^{r+1, r+1 / 2}$, with $\mathbf{n}^{r+1, r+1 / 2}=\frac{\mathbf{M}_{\mathbf{r}} \mathbf{M}_{\mathbf{r}+\mathbf{1}}}{\left\|\mathbf{M}_{\mathbf{r}} \mathbf{M}_{\mathbf{r}+\mathbf{1}}\right\|}$ and $\mathbf{n}^{r, r+1 / 2}=\frac{\mathbf{M}_{\mathbf{r}+\mathbf{1}} \mathbf{M}_{\mathbf{r}}}{\left\|\mathbf{M}_{\mathbf{r}} \mathbf{M}_{\mathbf{r}+\mathbf{1}}\right\|}$. Thus:

$$
\omega_{r+1 / 2}^{\prime}(t)=\frac{\partial \omega_{r+1 / 2}}{\partial d} \mathbf{U}_{r}(t) \cdot \mathbf{n}^{r, r+1 / 2}+\frac{\partial \omega_{r+1 / 2}}{\partial d} \mathbf{U}_{r+1}(t) \cdot \mathbf{n}^{r+1, r+1 / 2}+\frac{\partial \omega_{r+1 / 2}}{\partial l}\left(l_{j}^{r+1 / 2}\right)^{\prime}(t) .
$$

Then inserting (97) in (95), we obtain

$$
\left\{\begin{array}{l}
\mathbf{U}_{r+1 / 2}^{\text {defo }}(t)=\frac{h^{\prime}\left(\omega_{r-1 / 2}(t)\right)}{h\left(\omega_{r-1 / 2}(t)\right)} l_{j}^{r+1 / 2} \mathbf{n}_{j}^{r+1 / 2} \\
\left(\frac{\partial \omega_{r+1 / 2}}{\partial d} \mathbf{U}_{r}(t) \cdot \mathbf{n}^{r, r+1 / 2}+\frac{\partial \omega_{r+1 / 2}}{\partial d} \mathbf{U}_{r+1}(t) \cdot \mathbf{n}^{r+1, r+1 / 2}+\frac{\partial \omega_{r+1 / 2}}{\partial l}\left(l_{j}^{r+1 / 2}\right)^{\prime}(t)\right) .
\end{array}\right.
$$

Next inserting (98) in (93), we obtain

$$
\left\{\begin{aligned}
\frac{d}{d t}\left|C_{j}(t)\right|= & \sum_{r}\left(\tilde{\mathbf{C}}_{j}^{r, \omega(t)}+\frac{\partial \omega_{r+1 / 2}}{\partial d} \frac{h^{\prime}\left(\omega_{r+1 / 2}(t)\right)}{h\left(\omega_{r+1 / 2}(t)\right)} \mathbf{n}^{r, r+1 / 2}\left(\tilde{\mathbf{C}}_{j}^{r+1 / 2, \omega(t)}, l_{j}^{r+1 / 2} \mathbf{n}_{j}^{r+1 / 2}\right)\right. \\
& \left.+\frac{\partial \omega_{r-1 / 2}}{\partial d} \frac{h^{\prime}\left(\omega_{r-1 / 2}(t)\right)}{h\left(\omega_{r-1 / 2}(t)\right)} \mathbf{n}^{r, r-1 / 2}\left(\tilde{\mathbf{C}}_{j}^{r-1 / 2, \omega(t)}, l_{j}^{r-1 / 2} \mathbf{n}_{j}^{r-1 / 2}\right)\right) \cdot \mathbf{U}_{r}(t) \\
& +\sum_{r+1 / 2} \tilde{\mathbf{C}}_{j}^{r+1 / 2, \omega(t)} \cdot\left(U_{r+1 / 2}^{d i s p}+\frac{\partial \omega_{r+1 / 2}}{\partial l}\left(l_{j}^{r+1 / 2}\right)^{\prime}(t) \frac{h^{\prime}\left(\omega_{r+1 / 2}(t)\right)}{h\left(\omega_{r+1 / 2}(t)\right)} l_{j}^{r+1 / 2} \mathbf{n}_{j}^{r+1 / 2}\right) .
\end{aligned}\right.
$$

Finally using $\tilde{\mathbf{C}}_{j}^{r+1 / 2, \omega(t)} \cdot\left(\left(l_{j}^{r+1 / 2}\right)^{\prime}(t) \mathbf{n}_{j}^{r+1 / 2}\right)=\tilde{\mathbf{C}}_{j}^{r+1 / 2, \omega(t)} \cdot\left(\mathbf{U}_{r+1 / 2}^{\text {disp }}-\frac{1}{2}\left(\mathbf{U}_{r}+\mathbf{U}_{r+1}\right)\right)$ since $\dot{\mathbf{n}}_{j}^{r+1 / 2} \cdot \mathbf{n}_{j}^{r+1 / 2}=$ 0 , we obtain

$$
\frac{d}{d t}\left|C_{j}(t)\right|=\sum_{r} \mathbf{D}_{j}^{r, \omega(t)} \cdot \mathbf{U}_{r}(t)+\sum_{r+1 / 2} \mathbf{D}_{j}^{r+1 / 2, \omega(t)} \cdot \mathbf{U}_{r+1 / 2}^{d i s p}
$$

with

$$
\left\{\begin{aligned}
\mathbf{D}_{j}^{r, \omega(t)}= & \tilde{\mathbf{C}}_{j}^{r, \omega(t)} \\
& +\frac{\partial \omega_{r+1 / 2}}{\partial d} \frac{h^{\prime}\left(\omega_{r+1 / 2}(t)\right)}{h\left(\omega_{r+1 / 2}(t)\right)} \mathbf{n}^{r, r+1 / 2}\left(\tilde{\mathbf{C}}_{j}^{r+1 / 2, \omega(t)}, l_{j}^{r+1 / 2} \mathbf{n}_{j}^{r+1 / 2}\right) \\
& +\frac{\partial \omega_{r-1 / 2}}{\partial d} \frac{h^{\prime}\left(\omega_{r-1 / 2}(t)\right)}{h\left(\omega_{r-1 / 2}(t)\right)} \mathbf{n}^{r, r-1 / 2}\left(\tilde{\mathbf{C}}_{j}^{r-1 / 2, \omega(t)}, l_{j}^{r-1 / 2} \mathbf{n}_{j}^{r-1 / 2}\right) \\
& -\frac{1}{2} \frac{\partial \omega_{r-1 / 2}}{\partial l} \frac{h^{\prime}\left(\omega_{r-1 / 2}(t)\right)}{h\left(\omega_{r-1 / 2}(t)\right)} \tilde{\mathbf{C}}_{j}^{r-1 / 2, \omega(t)} l_{j}^{r-1 / 2} \\
& -\frac{1}{2} \frac{\partial \omega_{r+1 / 2}}{\partial l} \frac{h^{\prime}\left(\omega_{r+1 / 2}(t)\right)}{h\left(\omega_{r+1 / 2}(t)\right)} \tilde{\mathbf{C}}_{j}^{r+1 / 2, \omega(t)} l_{j}^{r+1 / 2}
\end{aligned}\right.
$$

and

$$
\mathbf{D}_{j}^{r+1 / 2, \omega(t)}=\tilde{\mathbf{C}}_{j}^{r+1 / 2, \omega(t)}\left(1+\frac{h^{\prime}\left(\omega_{r+1 / 2}(t)\right)}{h\left(\omega_{r+1 / 2}(t)\right)} \frac{\partial \omega_{r+1 / 2}}{\partial l} l_{j}^{r+1 / 2}\right) .
$$




\section{Remark 10.}

$$
\left\{\begin{array}{l}
\text { For each cell: } \quad \sum_{r} \mathbf{D}_{j}^{r, \omega(t)}+\sum_{r+\frac{1}{2}} \mathbf{D}_{j}^{r+1 / 2, \omega(t)}=0, \\
\text { for each internal node }: \sum_{j} \mathbf{D}_{j}^{r, \omega(t)}=0, \\
\text { for two adjacent cells } j \text { and } k: \mathbf{D}_{j}^{r+1 / 2, \omega(t)}+\mathbf{D}_{k}^{r+1 / 2, \omega(t)}=0 .
\end{array}\right.
$$

The two assertions can be demonstrated using:

$$
\left\{\begin{array}{l}
\text { For each cell : } \quad \sum_{r} \tilde{\mathbf{C}}_{j}^{r, \omega(t)}+\sum_{r+\frac{1}{2}} \tilde{\mathbf{C}}_{j}^{r+1 / 2, \omega(t)}=0, \\
\text { for each internal node }: \sum_{j} \tilde{\mathbf{C}}_{j}^{r, \omega(t)}=0, \\
\text { for two adjacent cells } \mathrm{j} \text { and } \mathrm{k}: \tilde{\mathbf{C}}_{j}^{r+1 / 2, \omega(t)}+\tilde{\mathbf{C}}_{k}^{r+1 / 2, \omega(t)}=0 .
\end{array}\right.
$$

and $\frac{\partial \omega}{\partial l}(-l)=-\frac{\partial \omega}{\partial l}(l)$ because of the parity of $\omega$ as a function of $l$ and $\mathbf{n}^{r, r+1 / 2}=-\mathbf{n}^{r+1, r+1 / 2}$.

Remark 11. (1) $\omega_{r+1 / 2}(t)$ is implicitly given by the geometrical constraint to have a circle passing through the three points : $M_{r}, S_{r+1 / 2}, M_{r+1}$ see also equation (94).

(2) Note that differential equations (89)(118) and (94) are equivalent for continuous time.

Remark 12. If we treat the particular case where $\omega_{r+1 / 2}(t)$ is kept constant, we recover [GLACE CIRCLE]. Indeed, since $\mathbf{U}_{r+1 / 2}^{\text {defo }}(t)=0$, we have

or

$$
\frac{d}{d t}\left|C_{j}(t)\right|=\sum_{r} \tilde{\mathbf{C}}_{j}^{r, \omega} \cdot \mathbf{U}_{r}(t)+\sum_{r+1 / 2} \tilde{\mathbf{C}}_{j}^{r+1 / 2, \omega} \cdot\left(\frac{1}{2}\left(\mathbf{U}_{r}(t)+\mathbf{U}_{r+1}(t)\right)+\mathbf{l}_{j}^{r+1 / 2 \mathbf{n}_{j}^{r+1 / 2}}\right),
$$

$$
\frac{d}{d t}\left|C_{j}(t)\right|=\sum_{r}\left(\tilde{\mathbf{C}}_{j}^{r, \omega}+\frac{1}{2}\left(\tilde{\mathbf{C}}_{j}^{r+1 / 2, \omega}+\tilde{\mathbf{C}}_{j}^{r-1 / 2, \omega}\right)\right) \cdot \mathbf{U}_{r}(t)+\sum_{r+1 / 2} \tilde{\mathbf{C}}_{j}^{r+1 / 2, \omega} \cdot\left(l_{j}^{r+1 / 2}\right)^{\prime}(t) \mathbf{n}_{j}^{r+1 / 2},
$$

but from (97) since $\omega_{r+1 / 2}^{\prime}(t)=0$ we have

We obtain

$$
\left\{\begin{aligned}
\left(l_{j}^{r+1 / 2}\right)^{\prime}(t) & =-\frac{\frac{\partial \omega_{r+1 / 2}}{\partial d}}{\frac{\partial \omega_{r+1 / 2}}{\partial l}} \mathbf{U}_{r}(t) \cdot \mathbf{n}^{r, r+1 / 2}-\frac{\frac{\partial \omega_{r+1 / 2}}{\partial d}}{\frac{\partial \omega_{r+1 / 2}}{\partial l}} \mathbf{U}_{r+1}(t) \cdot \mathbf{n}^{r+1, r+1 / 2} \\
& =\frac{l_{j}^{r+1 / 2}}{d_{r+1 / 2}}\left(\mathbf{U}_{r}(t) \cdot \mathbf{n}^{r, r+1 / 2}+\mathbf{U}_{r+1}(t) \cdot \mathbf{n}^{r+1, r+1 / 2}\right) .
\end{aligned}\right.
$$

with

$$
\frac{d}{d t}\left|C_{j}(t)\right|=\sum_{r} \mathbf{D}_{j}^{r, \omega} \cdot \mathbf{U}_{r}(t)
$$

$$
\left\{\begin{aligned}
\mathbf{D}_{j}^{r, \omega}= & \tilde{\mathbf{C}}_{j}^{r, \omega}+\frac{1}{2}\left(\mathbf{C}_{j}^{r+1 / 2, \omega}+\tilde{\mathbf{C}}_{j}^{r-1 / 2, \omega}\right) \\
& +\frac{l_{j}^{r+1 / 2}}{d_{r+1 / 2}} \mathbf{n}^{r, r+1 / 2}\left(\tilde{\mathbf{C}}_{j}^{r+1 / 2, \omega}, \mathbf{n}_{j}^{r+1 / 2}\right)+\frac{l_{j}^{r-1 / 2}}{d_{r-1 / 2}} \mathbf{n}^{r, r-1 / 2}\left(\tilde{\mathbf{C}}_{j}^{r-1 / 2, \omega}, \mathbf{n}_{j}^{r-1 / 2}\right) .
\end{aligned}\right.
$$

But we have

$$
\left\{\begin{array}{l}
\tilde{\mathbf{C}}_{j}^{r, \omega}+\frac{1}{2}\left(\tilde{\mathbf{C}}_{j}^{r+1 / 2, \omega}+\tilde{\mathbf{C}}_{j}^{r-1 / 2, \omega}\right)= \\
\mathbf{C}_{j}^{r}+\frac{l_{j}^{r+1 / 2}}{d_{r+1 / 2}} \mathbf{n}^{r, r+1 / 2}\left(\tilde{\mathbf{C}}_{j}^{r+1 / 2, \omega}, \mathbf{n}_{j}^{r+1 / 2}\right)+\frac{l_{j}^{r-1 / 2}}{d_{r-1 / 2}} \mathbf{n}^{r, r-1 / 2}\left(\tilde{\mathbf{C}}_{j}^{r-1 / 2, \omega}, \mathbf{n}_{j}^{r-1 / 2}\right)
\end{array}\right.
$$

with $\mathbf{C}_{j}^{r}$ the classical polygonal normal vector to the node $r$ since $\left(\tilde{\mathbf{C}}_{j}^{r+1 / 2, \omega}, \mathbf{n}_{j}^{r+1 / 2}\right)=\frac{h\left(\omega_{r+1 / 2}\right)}{2}$, becomes 


$$
\mathbf{D}_{j}^{r, \omega}=\mathbf{C}_{j}^{r}+\frac{l_{j}^{r+1 / 2}}{d_{r+1 / 2}} h\left(\omega_{r+1 / 2}\right) \mathbf{M}_{\mathbf{r}+\mathbf{1}} \mathbf{M}_{\mathbf{r}}+\frac{l_{j}^{r-1 / 2}}{d_{r-1 / 2}} h\left(\omega_{r-1 / 2}\right) \mathbf{M}_{\mathbf{r}-\mathbf{1}} \mathbf{M}_{\mathbf{r}}
$$

which can be put in a simpler form analog to [GLACE CIRCLE]

$$
\begin{aligned}
& \qquad \mathbf{D}_{j}^{r, \omega}=\mathbf{C}_{j}^{r}+2 \operatorname{sign}\left(l_{j}^{r+1 / 2}\right) \alpha_{r+1 / 2} \mathbf{M}_{\mathbf{r}+\mathbf{1}} \mathbf{M}_{\mathbf{r}}+2 \operatorname{sign}\left(l_{j}^{r-1 / 2}\right) \alpha_{r-1 / 2} \mathbf{M}_{\mathbf{r}-\mathbf{1}} \mathbf{M}_{\mathbf{r}}, \\
& \text { with } \alpha_{r+1 / 2}=\frac{\frac{\theta}{2}-\sin \left(\frac{\theta}{2}\right) \cos \left(\frac{\theta}{2}\right)}{4 \sin ^{2}\left(\frac{\theta}{2}\right)} .
\end{aligned}
$$

\section{Extension of GLACE Hydrodynamic scheme on CELls With Deforming CIRCULAR EDGES}

In this section, we give an extension of polygonal GLACE hydrodynamic scheme [GLACE] to meshes with deforming circular edges (1):

$$
\left\{\begin{array}{l}
D_{t} \int_{C(t)} 1 d v-\int_{\partial C(t)} \mathbf{U} \cdot \hat{\mathbf{N}} d s=0, \\
D_{t} \int_{C(t)} \rho d v=0 \\
D_{t} \int_{C(t)} \rho \mathbf{U} d v+\int_{\partial C(t)} P \cdot \hat{\mathbf{N}} d s=0 \\
D_{t} \int_{C(t)} \rho E d v+\int_{\partial C(t)} P \mathbf{U} \cdot \hat{\mathbf{N}} d s=0 .
\end{array}\right.
$$

If we denote $M(t)$ the mass of cell $C(t)\left(M(t)=\int_{C(t)} \rho d v\right)$ which is constant, the semi-discrete $(\omega(t), q=$ 1/2)-GLACE scheme on cells with deforming circular edges writes:

$$
\left\{\begin{array}{l}
M_{j} \tau_{j}^{\prime}(t)=\sum_{r}\left(\mathbf{D}_{j}^{r, \omega(t)}, \mathbf{U}_{r}\right)+\sum_{r+1 / 2}\left(\mathbf{D}_{j}^{r+1 / 2, \omega(t)}, \mathbf{U}_{r+1 / 2}\right), \quad \tau_{j}(t)=\frac{1}{\rho_{j}(t)}, \\
M_{j} \mathbf{U}_{j}^{\prime}(t)=-\sum_{r} \mathbf{D}_{j}^{r, \omega(t)} p_{j}^{r}-\sum_{r+1 / 2} \mathbf{D}_{j}^{r+1 / 2, \omega(t)} p_{j}^{r+1 / 2} \\
M_{j} E_{j}^{\prime}(t)=-\sum_{r}\left(\mathbf{D}_{j}^{r, \omega(t)}, \mathbf{U}_{r}\right) p_{j}^{r}-\sum_{r+1 / 2}\left(\mathbf{D}_{j}^{r+1 / 2, \omega(t)}, \mathbf{U}_{r+1 / 2}\right) p_{j}^{r+1 / 2}
\end{array}\right.
$$

where $\mathbf{D}_{j}^{r, \omega(t)}$ and $\mathbf{D}_{j}^{r+1 / 2, \omega(t)}$ are vectors computed in (100) (101). In order to determine $\mathbf{U}_{r}$ and $\mathbf{U}_{r+1 / 2}$ and the corresponding pressure $p_{j}^{r}$ and $p_{j}^{r+1 / 2}$, we apply the same techniques as in [GLACE] for nodes and shoulder points. The Riemann invariant is discretized:

(1) for nodes $r$, onto $\mathbf{D}_{j}^{r, \omega(t)}$ direction:

$$
p_{j}^{r}-p_{j}+\alpha_{j}^{r}\left(\mathbf{U}_{r}-\mathbf{U}_{j}, \mathbf{n}_{j}^{r, \omega(t)}\right)=0,
$$

where $\mathbf{n}_{j}^{r, \omega(t)}=\frac{\mathbf{D}_{j}^{r, \omega(t)}}{\left|\mathbf{D}_{j}^{r, \omega(t)}\right|}$ see (99) (100).

(2) for shoulder points $r+1 / 2$ of edges, onto $\mathbf{D}_{j}^{r+1 / 2, \omega(t)}$ direction:

$$
p_{j}^{r+1 / 2}-p_{j}+\alpha_{j}^{r+1 / 2}\left(\mathbf{U}_{r+1 / 2}-\mathbf{U}_{j}, \mathbf{n}_{j}^{r+1 / 2}\right)=0,
$$

with $\mathbf{n}_{j}^{r+1 / 2}=\frac{\mathbf{D}_{j}^{r+1 / 2, \omega(t)}}{\left|\mathbf{D}_{j}^{r+1 / 2, \omega(t)}\right|}$.

Then the speed and the pressure are entirely determined once $\mathbf{U}_{r}$ are computed.

Here $\alpha_{j}^{r}$ and $\alpha_{j}^{r+1 / 2}$ in (112) are the acoustic impedance $\rho_{j} c_{j}\left(c\right.$ is the sound speed $c^{2}=\left.\frac{\partial P}{\partial \rho}\right|_{S}$, and $\mathrm{S}$ the entropy) of the cell $C_{j} . \alpha_{j}^{r+1 / 2}$ remains to be fixed. To construct the solver we also enforce the following conservation condition:

- Around internal nodes:

$$
\sum_{j} p_{j}^{r} \mathbf{D}_{j}^{r, \omega(t)}=\mathbf{0} .
$$


To solve (111)(113), we consider the following two by two linear systems and obtain the velocities at each node:

$$
\left\{\begin{array}{l}
A_{r} \mathbf{U}_{r}=\mathbf{b}_{r}, \quad \text { with } \\
A_{r}=\sum_{j} \rho_{j} c_{j} \frac{\mathbf{D}_{j}^{r, \omega(t)} \otimes \mathbf{D}_{j}^{r, \omega(t)}}{\left|\mathbf{D}_{j}^{r, \omega(t)}\right|}, \\
b_{r}=\sum_{j} \mathbf{D}_{j}^{r, \omega(t)} p_{j}+\rho_{j} c_{j} \frac{\mathbf{D}_{j}^{r, \omega(t)} \otimes \mathbf{D}_{j}^{r, \omega(t)}}{\left|\mathbf{D}_{j}^{r, \omega(t)}\right|} \mathbf{U}_{j} .
\end{array}\right.
$$

This system is always invertible whatever the weight value for edges impinging at node $r$. Once the speeds are computed by (114) the pressure $p_{j}^{r}$ in (110) are computed using (111).

- Around shoulder point of internal edges:

$$
\begin{aligned}
& \sum_{j} p_{j}^{r+1 / 2} \mathbf{D}_{j}^{r+1 / 2, \omega(t)}=\mathbf{0}, \quad \text { which is equivalent to } \\
& p_{j}^{r+1 / 2} \mathbf{D}_{j}^{r+1 / 2, \omega(t)}+p_{k}^{r+1 / 2} \mathbf{D}_{k}^{r+1 / 2, \omega(t)}=\mathbf{0} .
\end{aligned}
$$

Hence in this case, thanks to the relation $\mathbf{D}_{k}^{r+1 / 2, \omega(t)}+\mathbf{D}_{j}^{r+1 / 2, \omega(t)}=0$ (see (102)), we obtain the continuity of pressure at shoulder point:

$$
p_{j}^{r+1 / 2}=p_{k}^{r+1 / 2} .
$$

We then apply the one dimensional Riemann solver that gives both normal speed and continuous pressure (see (116)):

$$
\left\{\begin{array}{l}
\mathbf{U}_{r+1 / 2} \cdot \mathbf{n}_{j}^{r+1 / 2}=\frac{\rho_{j} c_{j} \mathbf{U}_{\mathbf{j}} \cdot \mathbf{n}_{\mathbf{j}}^{\mathbf{r}+\mathbf{1} / \mathbf{2}}+\rho_{k} c_{k} \mathbf{U}_{\mathbf{k}} \cdot \mathbf{n}_{\mathbf{j}}^{\mathbf{r}+\mathbf{1} / \mathbf{2}}}{\rho_{j} c_{j}+\rho_{k} c_{k}}+\frac{1}{\rho_{j} c_{j}+\rho_{k} c_{k}}\left(p_{j}-p_{k}\right) \\
p^{r+1 / 2}=\frac{\rho_{j} c_{j} p_{j}+\rho_{k} c_{k} p_{k}}{\rho_{j} c_{j}+\rho_{k} c_{k}}+\frac{\rho_{j} c_{j} \rho_{k} c_{k}}{\rho_{j} c_{j}+\rho_{k} c_{k}}\left(\mathbf{U}_{\mathbf{j}}-\mathbf{U}_{\mathbf{k}}\right) \cdot \mathbf{n}_{\mathbf{j}}^{\mathbf{r}+\mathbf{1} / \mathbf{2}}
\end{array}\right.
$$

which gives

$$
\left(l_{j}^{r+1 / 2}\right)^{\prime}(t)=\mathbf{U}_{r+1 / 2} \cdot \mathbf{n}_{j}^{r+1 / 2}-\frac{1}{2}\left(\mathbf{U}_{r}(t)+\mathbf{U}_{r+1}(t)\right) \cdot \mathbf{n}_{j}^{r+1 / 2} .
$$

Remark 13. In this case, the tangential part of the speed is unequivocally defined by:

$$
\mathbf{U}_{r+1 / 2} \cdot \tau_{j}^{r+1 / 2}=\frac{1}{2}\left(\mathbf{U}_{r}(t)+\mathbf{U}_{r+1}(t)\right) \cdot \tau_{j}^{r+1 / 2}+l_{j}^{r+1 / 2}(t) \mathbf{n}_{j}{ }^{r+1 / 2} \cdot \tau_{j}^{r+1 / 2}
$$

where $\tau_{j}^{r+1 / 2} \cdot \mathbf{n}_{j}^{r+1 / 2}=0$.

Remark 14. From these equations, we can deduce an equation for the evolution of edge's curvature.

Noting $R_{r+1 / 2}$ the radius of the edge $r+1 / 2$ (see Figure (3)), since $\frac{d_{r+1 / 2}}{2 R_{r+1 / 2}}=\sin \left(\frac{\theta}{2}\right)$, we have $\frac{1}{R_{r+1 / 2}}=\frac{2 \sqrt{1-\omega_{r+1 / 2}(t)^{2}}}{d_{r+1 / 2}}$.

Then using

we obtain

$$
\omega_{r+1 / 2}^{\prime}(t)=\frac{\partial \omega_{r+1 / 2}(t)}{\partial d} d_{r+1 / 2}^{\prime}(t)+\frac{\partial \omega_{r+1 / 2}(t)}{\partial l}\left(l_{j}^{r+1 / 2}\right)^{\prime}(t)
$$

$$
\left\{\begin{array}{cl}
\left(\frac{1}{R_{r+1 / 2}}\right)^{\prime}(t)= & -2 d_{r+1 / 2}^{\prime}(t)\left(\frac{\omega_{r+1 / 2}(t)}{\sqrt{1-\omega_{r+1 / 2}(t)^{2}}} \frac{1}{d_{r+1 / 2}} \frac{\partial \omega_{r+1 / 2}(t)}{\partial d}+\frac{\sqrt{1-\omega_{r+1 / 2}(t)^{2}}}{\left(d_{r+1 / 2}\right)^{2}}\right) \\
& -2\left(l_{j}^{r+1 / 2}\right)^{\prime}(t)\left(\frac{\omega_{r+1 / 2}(t)}{\sqrt{1-\omega_{r+1 / 2}(t)^{2}}} \frac{1}{d_{r+1 / 2}} \frac{\partial \omega_{r+1 / 2}(t)}{\partial l}\right) .
\end{array}\right.
$$


Then noting that $d_{r+1 / 2}^{\prime}(t)=\mathbf{U}_{r}(t) \cdot \mathbf{n}^{r, r+1 / 2}+\mathbf{U}_{r+1}(t) \cdot \mathbf{n}^{r+1, r+1 / 2}$, we obtain an expression for the derivative of the curvature as a function of $\mathbf{U}_{r}(t), \mathbf{U}_{r+1}(t)$ and $\left(l_{j}^{r+1 / 2}\right)^{\prime}(t)$.

\section{CONCLUSiON}

In this paper, we have presented an extension of the centered Lagrangian $\omega$-EUCCLHYD scheme to new type of cells whose edges are conicals. We also have proposed a variant where the control points are replaced by points located on the edges excluding the two endpoints. At this stage, the conic segments are not allowed to deform ( $\omega$ are constant in time). In a second step, by computing the time derivative of the cell volume allowing the deformation of its edges, we have found a vectorial velocity describing this new behaviour. In our case, this edge velocity is uniquely characterized by the scalar parameter $\omega$. Finding an equation for $\omega(t)$ is still an opened question but for the special case when the conic segments are circles, by using this formalism, we were able to express $\omega^{\prime}(t)$ as a function of the velocities of the two endpoints and the arc midpoint. As a consequence, we have derived an equation for the curvature evolution of the circle segments. Then, we were able to propose an extension of the GLACE scheme to these cells. This new scheme can be seen as an extension of a previous work [GLACE CIRCLE] where $\omega$ was kept constant.

\section{FUture WORKS}

The coding of the last scheme is in progress. Numerical tests will follow. A particular issue will be the symmetry preservation for a one-dimensional polar radial flow. In the context of staggered schemes for cells with circle segments [MARSHA2], we think that the equation for the curvature evolution can allow to deal with unstructured meshes. In the general case, we think to link the evolution of $\omega(t)$ with the deformation tensors at the two endpoints. It leads to an expression of the time derivative of the cell volume in function of nodes not necessarily belonging to it. This work is in progress.

\section{REFERENCES}

[DUVAUT] G. Duvaut, Mécanique des milieux continus, Coll. Math. appl. pour la maîtrise, MASSON 1990.

[WAGUSEDE] Wang Guojin, T.W. Sederberg, Computing areas bounded by rational Bézier curves, CADDM, Vol 4, No. 2, pp. 18-27, September 1994.

[WAGU] Wang Guojin, Computing integral values involving nurbs curves, Jour. of Software, Vol 7, No. 9, pp. 542-546, September 1996.

[LIGACH] Ming Li, Xiao-Shan Gao, Shang-Ching Chou, Quadratic approximation to plane parametric curves and its application in approximate implicitization, Visual Comput., Vol 22, pp. 906-917, 2006.

[GLACE2D] B. Després, C. Mazeran., Lagrangian gas dynamics in two dimensions and lagrangian systems. Arch. Rational Mech. Anal., Vol 178, pp 327-372, 2005.

[DESLABSTAB] B. Després, E. Labourasse, Stabilization of the mesh for Lagrangian computations, http://multimat2011.celia.u-bordeaux1.fr/Multimat2011/Thursday_AM/Despres.pdf, Multi-Mat Conference, Arcachon September 2011.

[GLACE] G. Carre, S. Del Pino, B. Després, E. Labourasse, A cell-centered Lagrangian hydrodynamics scheme on general unstructured meshes in arbitrary dimension, Jour. Comp. Physic., Vol 228,pp 5160-5183, 2009.

[EUCCLHYD] P.H. Maire, R. Abgrall, J. Breil, J. Ovadia, A cell-centered Lagrangian scheme for two-dimensional compressible flow problems, SIAM J.Sci.Comput., Vol 29,pp 1781-1824, 2007.

[EQU] A. Chorin, J. Marsden, A Mathematical Introduction to Fluid Mechanics., Springer Verlag, 1992.

[GLACE CIRCLE] A. Claisse, B. Després, E. Labourasse, F. Ledoux, A new exceptional point method with application to cell-centered Lagrangian schemes and curved meshes submitted to J.Comp.Phys 2011.

[FEV] S. Del Pino, A curvilinear finite-volume method to solve compressible gas dynamics in semi-Lagrangian coordinates, CRAS, Vol 348, num 17-18, pp. 1027-1032, 2010.

[RIB] V. Dobrev, T. Ellis, T. Kolev, R. Rieben, Energy conserving finite element discretizations of Lagrangian hydrodynamics. Part 1: Theoretical framework, Downloadable presentation of Multimat'09 conference.

[BD] B. Després, Weak consistency of the cell centered Lagrangian GLACE scheme on general meshes in any dimension, Comp. Meth. Appl. Mech. Engr, 199, pp. 2669-2679, 2010.

[SHUSHEN] J. Cheng, C.W. Shu, A third order Conservative Lagrangian type scheme on curvilinear meshes for the compressible Euler equations, comm. on comput. phys., vol 4, no5, pp 1008-1024, 2008.

[HDRPHMAIRE] P.-H. Maire, Contribution to the numerical modeling of Inertial Confinement Fusion. Habilitation à Diriger des Recherches. Bordeaux University 2011. 
[CEMRACS10] B. Boutin, E. Deriaz, Ph. Hoch, P. Navaro, Extension of ALE methodology to unstructured conical meshes, ESAIM: PROCEEDINGS, October 2011, Vol. 32, p. 31-55.

[GO++] J.D. Benamou, P. Hoch, GO++ : A modular lagrangian/eulerian software for hamilton jacobi equations of geometric optics type, M2AN, 36(5):883-905, 2002.

[MARSHA2] L.G. Margolin, M. Shashkov, Using a curvilinear grid to construct symmetry-preserving discretizations for Lagrangian gas dynamics, J. Comput. Phys., vol 149, number 2, pp 389-417, 1999.

[GODUNOV] S. K. Godunov, A. V. Zabrodin, M. Ya. Ivanov, et al., Résolution numérique des problèmes multidimensionnels de la dynamiques des gaz (Editions Mir, Moscou, 1979).

[POSITLA] B. Larrouturou, How to preserve the mass fractions positivity when computing compressible multi-component flows, J. Comput. Phys. 95 (1991), 59-84. 